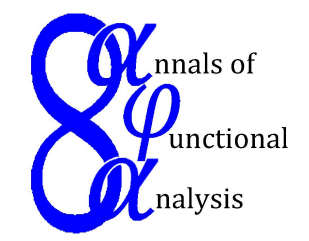

Ann. Funct. Anal. 6 (2015), no. 1, 59-83

http://doi.org/10.15352/afa/06-1-6

ISSN: 2008-8752 (electronic)

http://projecteuclid.org/afa

\title{
CAUCHY-TYPE MEANS AND EXPONENTIAL AND LOGARITHMIC CONVEXITY FOR SUPERQUADRATIC FUNCTIONS ON TIME SCALES
}

\author{
RABIA BIBI ${ }^{1 *}$, MARTIN BOHNER ${ }^{2}$ AND JOSIP PEČARIĆ ${ }^{3}$ \\ Communicated by K. Berenhaut
}

\begin{abstract}
In this paper, we define positive functionals by using the Jensen's inequality, converse of Jensen's inequality, and Jensen-Mercer's inequality on time scales for superquadratic functions. We give mean-value theorems and introduce related Cauchy-type means by using the functionals mentioned above and show the monotonicity of these means. We also show that these functionals are exponentially convex and give some applications of them by using the logconvexity and exponential convexity.
\end{abstract}

\section{INTRODUCTION}

Recently, the authors have presented time scales analogues of many important and well-known integral inequalities (Jensen's and its related inequalities) for convex functions [4] and superquadratic functions [6]. Also, in [5], Jensen's functionals are defined on time scales and several refinements and converses of Jensen's inequality are obtained by using properties of Jensen's functionals. Now, in this paper, we define Jensen type functionals on time scales for superquadratic functions and obtain several refinements, conversions and generalizations of Jensen's inequality on time scales for superquadratic functions. First we give some definitions and results which are used in the sequel.

Definition 1.1. A closed set $\emptyset \neq \mathbb{T} \subset \mathbb{R}$ is called a time scale. For $a, b \in \mathbb{T}$ with $a<b$, we denote $[a, b) \cap \mathbb{T}$ by $[a, b)_{\mathbb{T}}$. Let $t \in \mathbb{T}$. Then $\sigma: \mathbb{T} \rightarrow \mathbb{T}$ defined by

$$
\sigma(t)=\inf \{s \in \mathbb{T}: s>t\}
$$

Date: Received: Jan. 18, 2013; Accepted: Jun. 26, 2013.

* Corresponding author.

2010 Mathematics Subject Classification. Primary 26D15; Secondary 26E70, 28A25.

Key words and phrases. superquadratic functions, time scale, Jensen inequality, Cauchy means. 
is called the forward jump operator, and $\rho: \mathbb{T} \rightarrow \mathbb{T}$ defined by

$$
\rho(t)=\sup \{s \in \mathbb{T}: s<t\}
$$

is called the backward jump operator. Here we put inf $\emptyset=\sup \mathbb{T}$ and $\sup \emptyset=\inf \mathbb{T}$. If $\sigma(t)=t$, then $t$ is called right-dense, and if $\rho(t)=t$, then $t$ is called left-dense. A function $f: \mathbb{T} \rightarrow \mathbb{R}$ is called rd-continuous if it is continuous at all right-dense points in $\mathbb{T}$ and its left-sided limits are finite at all left-dense points in $\mathbb{T}$. The set of all such rd-continuous functions is denoted by $\mathrm{C}_{\mathrm{rd}}, \mathrm{C}_{\mathrm{rd}}(\mathbb{T})$, or $\mathrm{C}_{\mathrm{rd}}(\mathbb{T}, \mathbb{R})$. Finally, $f$ is called delta integrable if it has an antiderivative $F$ (i.e, $F^{\Delta}=f$ ), and then we define the delta integral by

$$
\int_{a}^{b} f(t) \Delta t=F(b)-F(a) .
$$

Theorem 1.2. Every rd-continuous function is delta integrable.

Theorem 1.3 (See $\left[4\right.$, Theorem 3.2]). Let $f, g \in \mathrm{C}_{\mathrm{rd}}\left([a, b)_{\mathbb{T}}, \mathbb{R}\right)$. Then

$$
\int_{a}^{b}(\alpha f(t)+\beta g(t)) \Delta t=\alpha \int_{a}^{b} f(t) \Delta t+\beta \int_{a}^{b} g(t) \Delta t \quad \text { for all } \quad \alpha, \beta \in \mathbb{R}
$$

and

$$
f(t) \geq 0 \quad \text { for all } \quad t \in[a, b)_{\mathbb{T}} \quad \text { implies } \quad \int_{a}^{b} f(t) \Delta t \geq 0 .
$$

Theorem 1.3 says that the time scales integral is an isotonic linear functional [8]. For further details of time scales theory, we refer to [7]. In the remainder of this section, we recall some relevant results from $[1,3,7]$.

Definition 1.4 (See [6, Definition 1.6]). A function $\Psi:[0, \infty) \rightarrow \mathbb{R}$ is called superquadratic if there exists a function $C:[0, \infty) \rightarrow \mathbb{R}$ such that

$$
\Psi(y)-\Psi(x)-\Psi(|y-x|) \geq C(x)(y-x) \text { for all } x, y \geq 0 .
$$

If for all $x, y>0$ with $x \neq y$, there is strict inequality in (1.1), then $\Psi$ is called strictly superquadratic.

Lemma 1.5 (See [6, Lemma 1.7]). Let $\Psi$ be a superquadratic function with $C(x)$ as in Definition 1.4. Then

(i) $\Psi(0) \leq 0$;

(ii) if $\Psi$ is differentiable at $x>0$ and $\Psi(0)=\Psi^{\prime}(0)=0$, then $C(x)=\Psi^{\prime}(x)$;

(iii) if $\Psi \geq 0$, then $\Psi$ is convex and $\Psi(0)=\Psi^{\prime}(0)=0$.

In the sequel, for any function $\Psi \in \mathrm{C}^{1}([0, \infty), \mathbb{R})$, we define an associated function $\bar{\Psi} \in \mathrm{C}^{1}((0, \infty), \mathbb{R})$ by

$$
\bar{\Psi}(x)=\frac{\Psi^{\prime}(x)}{x} \text { for all } \quad x>0 .
$$

Lemma 1.6 (See $\left[1\right.$, Lemma 1]). Let $\Psi \in \mathrm{C}^{1}([0, \infty), \mathbb{R})$ such that $\Psi(0) \leq 0$. If $\bar{\Psi}$ is increasing (strictly increasing) or $\Psi^{\prime}$ is superadditive (strictly superadditive), then $\Psi$ is superquadratic (strictly superquadratic). 
Lemma 1.7 (See $\left[1\right.$, Lemma 3]). Let $\Psi \in \mathrm{C}^{2}([0, \infty), \mathbb{R})$ be such that

$$
m_{1} \leq \frac{x \Psi^{\prime \prime}(x)-\Psi^{\prime}(x)}{x^{2}} \leq M_{1} \text { for all } x>0 .
$$

Let the functions $\Phi_{1}, \Phi_{2}$ be defined by

$$
\Phi_{1}(x)=\frac{M_{1} x^{3}}{3}-\Psi(x), \quad \Phi_{2}(x)=\Psi(x)-\frac{m_{1} x^{3}}{3} .
$$

Then $\overline{\Phi_{1}}, \overline{\Phi_{2}}$ are increasing. If also $\Psi(0)=0$ then $\Phi_{1}, \Phi_{2}$ are superquadratic.

Lemma 1.8. Let $s>0$ and $\Psi_{s}:[0, \infty) \rightarrow \mathbb{R}$ be defined by

$$
\Psi_{s}(x)= \begin{cases}\frac{x^{s}}{s(s-2)}, & s \neq 2, \\ \frac{x^{2}}{2} \log x, & s=2 .\end{cases}
$$

Then $\Psi_{s}$ is superquadratic, with the convention $0 \log 0:=0$.

Lemma 1.9. Let $s \in \mathbb{R}$ and $\varphi_{s}:[0, \infty) \rightarrow \mathbb{R}$ be defined by

$$
\varphi_{s}(x)= \begin{cases}\frac{s x e^{s x}-e^{s x}+1}{s^{3}}, & s \neq 0, \\ \frac{x^{3}}{3}, & s=0 .\end{cases}
$$

Then $\varphi_{s}$ is superquadratic.

Definition 1.10 (See [3, Definition 1]). A function $\Lambda:(a, b) \rightarrow \mathbb{R}$ is called exponentially convex if it is continuous and

$$
\sum_{i, j=1}^{n} v_{i} v_{j} \Lambda\left(x_{i}+x_{j}\right) \geq 0
$$

for all $n \in \mathbb{N}$ and all choices $v_{i} \in \mathbb{R}$ and $x_{i}+x_{j} \in(a, b), 1 \leq i, j \leq n$.

Proposition 1.11 (See [3, Proposition 1]). Let $\Lambda:(a, b) \rightarrow \mathbb{R}$. The following are equivalent:

(i) $\Lambda$ is exponentially convex.

(ii) $\Lambda$ is continuous and

$$
\sum_{i, j=1}^{n} v_{i} v_{j} \Lambda\left(\frac{x_{i}+x_{j}}{2}\right) \geq 0
$$

for all $n \in \mathbb{N}, v_{i} \in \mathbb{R}$ and $x_{i}+x_{j} \in(a, b), 1 \leq i, j \leq n$.

(iii) $\Lambda$ is continuous and

$$
\operatorname{det}\left[\Lambda\left(\frac{x_{i}+x_{j}}{2}\right)\right]_{i, j=1}^{m} \geq 0, \quad 1 \leq m \leq n
$$

for all $n \in \mathbb{N}$ and for every $x_{i} \in(a, b), i=1, \ldots, n$. 
Remark 1.12. Let $\Lambda:(a, b) \rightarrow(0, \infty)$ be an exponentially convex function. Then $\Lambda$ is also $\log$-convex, i.e., $\log \Lambda$ is convex. If $r, s, w \in(a, b)$ such that $r<s<w$, then

$$
[\Lambda(s)]^{w-r} \leq[\Lambda(r)]^{w-s}[\Lambda(w)]^{s-r} .
$$

Lemma 1.13 (See [1, Lemma 2]). Let $\Lambda:(a, b) \rightarrow(0, \infty)$ be a log-convex function. Then for any $r, l, v, w \in(a, b)$ such that $r \leq v, l \leq w, r \neq l, v \neq w$, we have

$$
\left(\frac{\Lambda(r)}{\Lambda(l)}\right)^{\frac{1}{r-l}} \leq\left(\frac{\Lambda(v)}{\Lambda(w)}\right)^{\frac{1}{v-w}}
$$

\section{Jensen-Type Inequalities}

In order to obtain our main results, we first recall from [6] Jensen's inequality and its converses on time scales for superquadratic functions.

Theorem 2.1 (Jensen's inequality [6, Theorem 2.5]). Let $a, b \in \mathbb{T}$. Suppose $f \in \mathrm{C}_{\mathrm{rd}}\left([a, b)_{\mathbb{T}},[0, \infty)\right)$ and $\Psi \in \mathrm{C}([0, \infty), \mathbb{R})$ is superquadratic. Then

$$
\Psi\left(\frac{\int_{a}^{b} f(t) \Delta t}{b-a}\right) \leq \frac{1}{b-a} \int_{a}^{b}\left[\Psi(f(u))-\Psi\left(\left|f(u)-\frac{\int_{a}^{b} f(t) \Delta t}{b-a}\right|\right)\right] \Delta u .
$$

Moreover, if $\Psi$ is strictly superquadratic, then strict inequality in (2.1) holds.

Theorem 2.2 (Jensen-Mercer inequality [6, Theorem 5.2]). Let $a, b \in \mathbb{T}$. Suppose $f \in \mathrm{C}_{\mathrm{rd}}\left([a, b)_{\mathbb{T}},[m, M]\right)$, where $0 \leq m<M<\infty$, and $\Psi \in \mathrm{C}([0, \infty), \mathbb{R})$ is superquadratic. Then

$$
\begin{aligned}
& (b-a) \Psi\left(m+M-\frac{1}{b-a} \int_{a}^{b} f(t) \Delta t\right) \\
& \quad \leq(b-a)(\Psi(m)+\Psi(M))-\int_{a}^{b} \Psi(f(t)) \Delta t-K,
\end{aligned}
$$

where

$$
\begin{aligned}
K=\frac{2}{M-m} \int_{a}^{b}[(f(t)-m) \Psi(M- & f(t))+(M-f(t)) \Psi(f(t)-m)] \Delta t \\
& +\int_{a}^{b} \Psi\left(\left|f(u)-\frac{1}{b-a} \int_{a}^{b} f(t) \Delta t\right|\right) \Delta u .
\end{aligned}
$$

Moreover, if $\Psi$ is strictly superquadratic, then strict inequality in (2.2) holds.

Theorem 2.3 (Converse Jensen inequality [6, Theorem 6.2]). Let $a, b \in \mathbb{T}$. Suppose $f \in \mathrm{C}_{\mathrm{rd}}\left([a, b)_{\mathbb{T}},[m, M]\right)$, where $0 \leq m<M<\infty$, and $\Psi \in \mathrm{C}([0, \infty), \mathbb{R})$ is superquadratic. Then

$$
\begin{aligned}
\int_{a}^{b} \Psi(f(t)) & \Delta t+R \\
& \leq \frac{M(b-a)-\int_{a}^{b} f(t) \Delta t}{M-m} \Psi(m)+\frac{\int_{a}^{b} f(t) \Delta t-m(b-a)}{M-m} \Psi(M),
\end{aligned}
$$


where

$$
R=\frac{1}{M-m} \int_{a}^{b}[(f(t)-m) \Psi(M-f(t))+(M-f(t)) \Psi(f(t)-m)] \Delta t .
$$

Moreover, if $\Psi$ is strictly superquadratic, then strict inequality in (2.3) holds.

Remark 2.4. The above three theorems hold for many other time scales integrals, such as Cauchy, Riemann, Lebesgue, multiple Riemann, and multiple Lebesgue delta, nabla, and diamond- $\alpha$ time scales integrals as we know that these integrals are isotonic linear functionals (see [4]).

Remark 2.5. Weighted version of Theorems 2.1, 2.2, and 2.3 also hold, i.e., we can take the weighted mean $\frac{\int_{a}^{b} k(t) f(t) \Delta t}{\int_{a}^{b} k(t) \Delta t}$ instead of $\frac{\int_{a}^{b} f(t) \Delta t}{b-a}$, where

$$
k \in \mathrm{C}_{\mathrm{rd}}\left([a, b)_{\mathbb{T}},[0, \infty)\right) \quad \text { is such that } \int_{a}^{b} k(t) \Delta t>0
$$

\section{Cauchy-Type Means}

Under the assumptions of Theorems 2.1, 2.2 and 2.3, we define functionals $\mathcal{J}_{\Psi}$, $\widetilde{\mathcal{J}}_{\Psi}$, and $\widehat{\mathcal{J}}_{\Psi}$ by

$$
\begin{aligned}
\mathcal{J}_{\Psi}=\int_{a}^{b}\left[\Psi(f(u))-\Psi\left(\left|f(u)-\frac{\int_{a}^{b} f(t) \Delta t}{b-a}\right|\right)\right] \Delta u \\
-(b-a) \Psi\left(\frac{\int_{a}^{b} f(t) \Delta t}{b-a}\right) \\
\widetilde{\mathcal{J}}_{\Psi}=(b-a)(\Psi(m)+\Psi(M))-\int_{a}^{b} \Psi(f(t)) \Delta t-K \\
-(b-a) \Psi\left(m+M-\frac{1}{b-a} \int_{a}^{b} f(t) \Delta t\right), \\
\widehat{\mathcal{J}}_{\Psi}=\frac{M(b-a)-\int_{a}^{b} f(t) \Delta t}{M-m} \Psi(m)+\frac{\int_{a}^{b} f(t) \Delta t-m(b-a)}{M-m} \Psi(M) \\
-\int_{a}^{b} \Psi(f(t)) \Delta t-R .
\end{aligned}
$$

From the inequalities (2.1), (2.2), and (2.3), it is clear that, subject to the relevant assumptions, $\mathcal{J}_{\Psi}, \widetilde{\mathcal{J}}_{\Psi}$, and $\widehat{\mathcal{J}}_{\Psi}$ are nonnegative.

Theorem 3.1. Let $a, b \in \mathbb{T}$. Suppose $f \in \mathrm{C}_{\mathrm{rd}}\left([a, b)_{\mathbb{T}},[0, \infty)\right)$ and $\Psi \in \mathrm{C}^{1}([0, \infty), \mathbb{R})$ is such that $\Psi(0)=0$ and $\bar{\Psi} \in \mathrm{C}^{1}((0, \infty), \mathbb{R})$. Then

$$
\mathcal{J}_{\Psi}=\frac{\varrho \Psi^{\prime \prime}(\varrho)-\Psi^{\prime}(\varrho)}{\varrho^{2}} \mathcal{J}_{\Psi_{3}}
$$

holds for some $\varrho>0$, provided that $\mathcal{J}_{\Psi_{3}} \neq 0$, where $\Psi_{3}$ is defined in (1.3). 
Proof. Define

$$
\psi_{*}:=\inf _{x \in(0, \infty)} \bar{\Psi}^{\prime}(x) \text { and } \quad \psi^{*}:=\sup _{x \in(0, \infty)} \bar{\Psi}^{\prime}(x)
$$

Case 1: Suppose

$$
\psi_{*}=\min _{x \in(0, \infty)} \bar{\Psi}^{\prime}(x) \quad \text { and } \quad \psi^{*}=\max _{x \in(0, \infty)} \bar{\Psi}^{\prime}(x) .
$$

Then

$$
\psi_{*} \leq \frac{x \Psi^{\prime \prime}(x)-\Psi^{\prime}(x)}{x^{2}} \leq \psi^{*} \text { for all } x>0 .
$$

Hence by Lemma 1.7, $\Phi_{1}$ and $\Phi_{2}$ defined in (1.2) are superquadratic. By Theorem 2.1, we have $\mathcal{J}_{\Phi_{1}}, \mathcal{J}_{\Phi_{2}} \geq 0$. Thus, since $\mathcal{J}_{\Phi_{1}}=\psi^{*} \mathcal{J}_{\Psi_{3}}-\mathcal{J}_{\Psi}$ and $\mathcal{J}_{\Phi_{2}}=\mathcal{J}_{\Psi}-\psi_{*} \mathcal{J}_{\Psi_{3}}$, we obtain

$$
\psi_{*} \mathcal{J}_{\Psi_{3}} \leq \mathcal{J}_{\Psi} \leq \psi^{*} \mathcal{J}_{\Psi_{3}}
$$

Now, (3.3) and (3.4) imply that there exists some $\varrho>0$ such that (3.2) holds.

Case 2: Suppose

$$
\psi_{*}=\min _{x \in(0, \infty)} \bar{\Psi}^{\prime}(x) \quad \text { and } \quad \psi^{*} \neq \max _{x \in(0, \infty)} \bar{\Psi}^{\prime}(x) .
$$

In this case, $\Phi_{1}$ is strictly superquadratic. Therefore $\mathcal{J}_{\Phi_{1}}>0$ and $\mathcal{J}_{\Phi_{2}} \geq 0$. Hence

$$
\psi_{*} \leq \frac{x \Psi^{\prime \prime}(x)-\Psi^{\prime}(x)}{x^{2}}<\psi^{*}
$$

and thus

$$
\psi_{*} \mathcal{J}_{\Psi_{3}} \leq \mathcal{J}_{\Psi}<\psi^{*} \mathcal{J}_{\Psi_{3}}
$$

Now, (3.5) and (3.6) imply that (3.2) holds for some $\varrho>0$.

Case 3: Suppose

$$
\psi_{*} \neq \min _{x \in(0, \infty)} \bar{\Psi}^{\prime}(x) \quad \text { and } \quad \psi^{*}=\max _{x \in(0, \infty)} \bar{\Psi}^{\prime}(x) .
$$

In this case, $\Phi_{2}$ is strictly superquadratic. The rest of the proof is analogous to the proof in Case 2.

Case 4: Suppose

$$
\psi_{*} \neq \min _{x \in(0, \infty)} \bar{\Psi}^{\prime}(x) \quad \text { and } \quad \psi^{*} \neq \max _{x \in(0, \infty)} \bar{\Psi}^{\prime}(x) .
$$

In this case, $\Phi_{1}$ and $\Phi_{2}$ both are strictly superquadratic. The rest of the proof is analogous to the proof in Case 2.

In the case where $\psi^{*}=\infty$ (i.e., $\bar{\Psi}^{\prime}$ is not bounded above) and $\psi_{*}$ exists, using just $\Phi_{2}$, we obtain

$$
\psi_{*} \leq \frac{x \Psi^{\prime \prime}(x)-\Psi^{\prime}(x)}{x^{2}}
$$

in the case of minimum, and strong inequality in the case where $\psi_{*}$ is infimum. The rest of the proof is as above. The remaining cases can be treated analogously. 
Theorem 3.2. Let $a, b \in \mathbb{T}$ and $f \in \mathrm{C}_{\mathrm{rd}}\left([a, b)_{\mathbb{T}},[0, \infty)\right)$ such that $\mathcal{J}_{\Psi_{3}} \neq 0$. Suppose $\Psi, \Phi \in \mathrm{C}^{1}([0, \infty), \mathbb{R})$ are such that $\Psi(0)=\Phi(0)=0$ and $\bar{\Psi}, \bar{\Phi} \in$ $\mathrm{C}^{1}((0, \infty), \mathbb{R})$. Then there exists some $\varrho>0$ such that

$$
\frac{\mathcal{J}_{\Psi}}{\mathcal{J}_{\Phi}}=\frac{\varrho \Psi^{\prime \prime}(\varrho)-\Psi^{\prime}(\varrho)}{\varrho \Phi^{\prime \prime}(\varrho)-\Phi^{\prime}(\varrho)}
$$

holds, provided that the denominators in (3.7) are nonzero.

Proof. Define $\chi \in \mathrm{C}^{1}([0, \infty), \mathbb{R})$ by

$$
\chi(x)=\mathcal{J}_{\Phi} \Psi(x)-\mathcal{J}_{\Psi} \Phi(x) \quad \text { for } \quad x \geq 0 .
$$

Then $\bar{\chi} \in \mathrm{C}^{1}((0, \infty), \mathbb{R}), \chi(0)=0$, and $\mathcal{J}_{\chi}=0$. Therefore, by using $\chi$ instead of $\Psi$ in Theorem 3.1, we obtain that there exists $\varrho>0$ such that

$$
0=\varrho \chi^{\prime \prime}(\varrho)-\chi^{\prime}(\varrho)=\mathcal{J}_{\Phi}\left(\varrho \Psi^{\prime \prime}(\varrho)-\Psi^{\prime}(\varrho)\right)-\mathcal{J}_{\Psi}\left(\varrho \Phi^{\prime \prime}(\varrho)-\Phi^{\prime}(\varrho)\right),
$$

from which (3.7) follows.

Remark 3.3. In Theorem 3.2, let

$$
\mathcal{G}(\varrho)=\frac{\varrho \Psi^{\prime \prime}(\varrho)-\Psi^{\prime}(\varrho)}{\varrho \Phi^{\prime \prime}(\varrho)-\Phi^{\prime}(\varrho)}
$$

and suppose $\mathcal{G}$ is invertible. Then we obtain another mean defined by

$$
\varrho=\mathcal{G}^{-1}\left(\frac{\mathcal{J}_{\Psi}}{\mathcal{J}_{\Phi}}\right)
$$

Theorem 3.4. Let $a, b \in \mathbb{T}$. Suppose $f \in \mathrm{C}_{\mathrm{rd}}\left([a, b)_{\mathbb{T}},[m, M]\right)$, where $0 \leq m<$ $M<\infty$, and $\Psi \in \mathrm{C}^{1}([0, \infty), \mathbb{R})$ is such that $\Psi(0)=0$ and $\bar{\Psi} \in \mathrm{C}^{1}((0, \infty), \mathbb{R})$. Then

$$
\widetilde{\mathcal{J}}_{\Psi}=\frac{\varrho \Psi^{\prime \prime}(\varrho)-\Psi^{\prime}(\varrho)}{\varrho^{2}} \widetilde{\mathcal{J}}_{\Psi_{3}}
$$

holds for some $\varrho>0$, provided that $\widetilde{\mathcal{J}}_{\Psi_{3}} \neq 0$.

Proof. The proof is analogous to the proof of Theorem 3.1, where, instead of using Theorem 2.1, we apply Theorem 2.2 to $\Phi_{1}$ and $\Phi_{2}$.

Theorem 3.5. Let $a, b \in \mathbb{T}$ and $f \in \mathrm{C}_{\mathrm{rd}}\left([a, b)_{\mathbb{T}},[m, M]\right)$, where $0 \leq m<M<\infty$, such that $\widetilde{\mathcal{J}}_{\Psi_{3}} \neq 0$. Suppose $\Psi, \Phi \in \mathrm{C}^{1}([0, \infty), \mathbb{R})$ are such that $\Psi(0)=\Phi(0)=0$ and $\bar{\Psi}, \bar{\Phi} \in \mathrm{C}^{1}((0, \infty), \mathbb{R})$. Then there exists some $\varrho>0$ such that

$$
\frac{\widetilde{\mathcal{J}}_{\Psi}}{\widetilde{\mathcal{J}}_{\Phi}}=\frac{\varrho \Psi^{\prime \prime}(\varrho)-\Psi^{\prime}(\varrho)}{\varrho \Phi^{\prime \prime}(\varrho)-\Phi^{\prime}(\varrho)}
$$

holds, provided that the denominators in (3.8) are nonzero.

Proof. The proof is analogous to the proof of Theorem 3.2, where, instead of using Theorem 3.1, we apply Theorem 3.4 to $\chi$. 
Remark 3.6. In Theorem 3.5, let

$$
\widetilde{\mathcal{G}}(\varrho)=\frac{\varrho \Psi^{\prime \prime}(\varrho)-\Psi^{\prime}(\varrho)}{\varrho \Phi^{\prime \prime}(\varrho)-\Phi^{\prime}(\varrho)}
$$

and suppose $\widetilde{\mathcal{G}}$ is invertible. Then we obtain another mean defined by

$$
\varrho=\widetilde{\mathcal{G}}^{-1}\left(\frac{\widetilde{\mathcal{J}}_{\Psi}}{\widetilde{\mathcal{J}}_{\Phi}}\right) .
$$

Theorem 3.7. Let $a, b \in \mathbb{T}$. Suppose $f \in \mathrm{C}_{\mathrm{rd}}\left([a, b)_{\mathbb{T}},[m, M]\right)$, where $0 \leq m<$ $M<\infty$, and $\Psi \in \mathrm{C}^{1}([0, \infty), \mathbb{R})$ is such that $\Psi(0)=0$ and $\Psi \in \mathrm{C}^{1}((0, \infty), \mathbb{R})$. Then

$$
\widehat{\mathcal{J}}_{\Psi}=\frac{\varrho \Psi^{\prime \prime}(\varrho)-\Psi^{\prime}(\varrho)}{\varrho^{2}} \widehat{\mathcal{J}}_{\Psi_{3}}
$$

holds for some $\varrho>0$, provided that $\widehat{\mathcal{J}}_{\Psi_{3}} \neq 0$.

Proof. The proof is analogous to the proof of Theorem 3.1, where, instead of using Theorem 2.1, we apply Theorem 2.3 to $\Phi_{1}$ and $\Phi_{2}$.

Theorem 3.8. Let $a, b \in \mathbb{T}$ and $f \in \mathrm{C}_{\mathrm{rd}}\left([a, b)_{\mathbb{T}},[m, M]\right)$, where $0 \leq m<M<\infty$, such that $\widehat{\mathcal{J}}_{\Psi_{3}} \neq 0$. Suppose $\Psi, \Phi \in \mathrm{C}^{1}([0, \infty), \mathbb{R})$ are such that $\Psi(0)=\Phi(0)=0$ and $\bar{\Psi}, \bar{\Phi} \in \mathrm{C}^{1}((0, \infty), \mathbb{R})$. Then there exists some $\varrho>0$ such that

$$
\frac{\widehat{\mathcal{J}}_{\Psi}}{\widehat{\mathcal{J}}_{\Phi}}=\frac{\varrho \Psi^{\prime \prime}(\varrho)-\Psi^{\prime}(\varrho)}{\varrho \Phi^{\prime \prime}(\varrho)-\Phi^{\prime}(\varrho)}
$$

holds, provided that the denominators in (3.9) are nonzero.

Proof. The proof is analogous to the proof of Theorem 3.2, where, instead of using Theorem 3.1, we apply Theorem 3.7 to $\chi$.

Remark 3.9. In Theorem 3.8, let

$$
\widehat{\mathcal{G}}(\varrho)=\frac{\varrho \Psi^{\prime \prime}(\varrho)-\Psi^{\prime}(\varrho)}{\varrho \Phi^{\prime \prime}(\varrho)-\Phi^{\prime}(\varrho)}
$$

and suppose $\widehat{\mathcal{G}}$ is invertible. Then we obtain another mean defined by

$$
\varrho=\widehat{\mathcal{G}}^{-1}\left(\frac{\widehat{\mathcal{J}}_{\Psi}}{\widehat{\mathcal{J}}_{\Phi}}\right) .
$$

\section{Generalized Means}

Definition 4.1 (See [5, Definition 3.1]). Let $a, b \in \mathbb{T}$. Let $\alpha \in \mathrm{C}(I, \mathbb{R})$ be strictly monotone, where $I \subset \mathbb{R}$ is an interval. If $f \in \mathrm{C}_{\mathrm{rd}}\left([a, b)_{\mathbb{T}}, I\right)$, then the generalized mean of $f$ is defined by

$$
\mathfrak{M}_{\alpha}(f)=\alpha^{-1}\left(\frac{\int_{a}^{b}(\alpha \circ f)(t) \Delta t}{b-a}\right),
$$

provided that (4.1) is well defined. 
Theorem 4.2. Let $a, b \in \mathbb{T}$ and $f \in \mathrm{C}_{\mathrm{rd}}\left([a, b)_{\mathbb{T}},[0, \infty)\right)$. Suppose, moreover, that $\alpha, \beta, \gamma \in \mathrm{C}^{2}([0, \infty), \mathbb{R})$ are strictly monotone such that

$$
\overline{\alpha \circ \gamma^{-1}}, \overline{\beta \circ \gamma^{-1}} \in \mathrm{C}^{1}((0, \infty), \mathbb{R}) \quad \text { and } \quad\left(\alpha \circ \gamma^{-1}\right)(0)=\left(\beta \circ \gamma^{-1}\right)(0)=0 .
$$

If

$\int_{a}^{b}\left((\gamma \circ f)^{3}(u)-\left|(\gamma \circ f)(u)-\frac{\int_{a}^{b}(\gamma \circ f)(t) \Delta t}{b-a}\right|^{3}\right) \Delta u-\frac{\left(\int_{a}^{b}(\gamma \circ f)(t) \Delta t\right)^{3}}{(b-a)^{2}} \neq 0$

then

$$
\begin{aligned}
\frac{\alpha\left(\mathfrak{M}_{\alpha}(f)\right)-\alpha\left(\mathfrak { M } _ { \alpha } \left(\gamma^{-1}(\mid\right.\right.}{\left.\left.\left.\beta(\gamma \circ f)-\gamma\left(\mathfrak{M}_{\gamma}(f)\right) \mid\right)\right)\right)-\alpha\left(\mathfrak{M}_{\gamma}(f)\right)} \\
=\frac{\gamma(\zeta)\left(\alpha^{\prime \prime}(\zeta) \gamma^{\prime}(\zeta)-\alpha^{\prime}(\zeta) \gamma^{\prime \prime}(\zeta)\right)-\alpha^{\prime}(\zeta)\left(\gamma^{\prime}(\zeta)\right)^{2}}{\gamma(\zeta)\left(\beta^{\prime \prime}(\zeta) \gamma^{\prime}(\zeta)-\beta^{\prime}(\zeta) \gamma^{\prime \prime}(\zeta)\right)-\beta^{\prime}(\zeta)\left(\gamma^{\prime}(\zeta)\right)^{2}}
\end{aligned}
$$

holds for some $\zeta \in f\left([a, b)_{\mathbb{T}}\right)$, provided that the denominators in (4.2) are nonzero.

Proof. Replace the functions $f, \Psi$ and $\Phi$ in Theorem 3.2 by $\gamma \circ f, \alpha \circ \gamma^{-1}$ and $\beta \circ \gamma^{-1}$, respectively, so there exists some $\varrho>0$ such that

$$
\begin{aligned}
& \quad \frac{\alpha\left(\mathfrak{M}_{\alpha}(f)\right)-\alpha\left(\mathfrak{M}_{\alpha}\left(\gamma^{-1}\left(\left|(\gamma \circ f)-\gamma\left(\mathfrak{M}_{\gamma}(f)\right)\right|\right)\right)\right)-\alpha\left(\mathfrak{M}_{\gamma}(f)\right)}{\beta\left(\mathfrak{M}_{\beta}(f)\right)-\beta\left(\mathfrak{M}_{\beta}\left(\gamma^{-1}\left(\left|(\gamma \circ f)-\gamma\left(\mathfrak{M}_{\gamma}(f)\right)\right|\right)\right)\right)-\beta\left(\mathfrak{M}_{\gamma}(f)\right)} \\
& =\frac{\varrho\left(\alpha^{\prime \prime}\left(\gamma^{-1}(\varrho)\right) \gamma^{\prime}\left(\gamma^{-1}(\varrho)\right)-\alpha^{\prime}\left(\gamma^{-1}(\varrho)\right) \gamma^{\prime \prime}\left(\gamma^{-1}(\varrho)\right)\right)-\alpha^{\prime}\left(\gamma^{-1}(\varrho)\right)\left(\gamma^{\prime}\left(\gamma^{-1}(\varrho)\right)\right)^{2}}{\varrho\left(\beta^{\prime \prime}\left(\gamma^{-1}(\varrho)\right) \gamma^{\prime}\left(\gamma^{-1}(\varrho)\right)-\beta^{\prime}\left(\gamma^{-1}(\varrho)\right) \gamma^{\prime \prime}\left(\gamma^{-1}(\varrho)\right)\right)-\beta^{\prime}\left(\gamma^{-1}(\varrho)\right)\left(\gamma^{\prime}\left(\gamma^{-1}(\varrho)\right)\right)^{2}}
\end{aligned}
$$

By putting $\gamma^{-1}(\varrho)=\zeta$, there exists some $\zeta \in f\left([a, b)_{\mathbb{T}}\right)$ such that $(4.2)$ holds.

Remark 4.3. In Theorem 4.2, let

$$
\mathcal{F}(\zeta)=\frac{\gamma(\zeta)\left(\alpha^{\prime \prime}(\zeta) \gamma^{\prime}(\zeta)-\alpha^{\prime}(\zeta) \gamma^{\prime \prime}(\zeta)\right)-\alpha^{\prime}(\zeta)\left(\gamma^{\prime}(\zeta)\right)^{2}}{\gamma(\zeta)\left(\beta^{\prime \prime}(\zeta) \gamma^{\prime}(\zeta)-\beta^{\prime}(\zeta) \gamma^{\prime \prime}(\zeta)\right)-\beta^{\prime}(\zeta)\left(\gamma^{\prime}(\zeta)\right)^{2}}
$$

and suppose $\mathcal{F}$ is invertible. Then, since $\zeta$ is in the image of $f$, we obtain a new mean defined by

$$
\mathcal{F}^{-1}\left(\frac{\alpha\left(\mathfrak{M}_{\alpha}(f)\right)-\alpha\left(\mathfrak{M}_{\alpha}\left(\gamma^{-1}\left(\left|(\gamma \circ f)-\gamma\left(\mathfrak{M}_{\gamma}(f)\right)\right|\right)\right)\right)-\alpha\left(\mathfrak{M}_{\gamma}(f)\right)}{\beta\left(\mathfrak{M}_{\beta}(f)\right)-\beta\left(\mathfrak{M}_{\beta}\left(\gamma^{-1}\left(\left|(\gamma \circ f)-\gamma\left(\mathfrak{M}_{\gamma}(f)\right)\right|\right)\right)\right)-\beta\left(\mathfrak{M}_{\gamma}(f)\right)}\right) .
$$

Definition 4.4 (See [5, Definition 3.4]). Let $a, b \in \mathbb{T}$ and $f \in \mathrm{C}_{\mathrm{rd}}\left([a, b)_{\mathbb{T}}, I\right)$, where $I \subset \mathbb{R}$ is an interval. If $r \in \mathbb{R}$, then the generalized power mean of $f$ is defined by

$$
\mathfrak{M}_{r}(f)= \begin{cases}\left(\frac{\int_{a}^{b} f^{r}(t) \Delta t}{b-a}\right)^{\frac{1}{r}}, & r \neq 0, \\ \exp \left(\frac{\int_{a}^{b} \log f(t) \Delta t}{b-a}\right), & r=0,\end{cases}
$$

provided that (4.3) is well defined. 
Corollary 4.5. Let $a, b \in \mathbb{T}$ and $f \in \mathrm{C}_{\mathrm{rd}}\left([a, b)_{\mathbb{T}}, I\right)$ be positive. Suppose $r, l, s>0$ are such that $r \neq l, r \neq 2 s, l \neq 2 s$, and

$$
\int_{a}^{b}\left(f^{3 s}(u)-\left|f^{s}(u)-\frac{\int_{a}^{b} f^{s}(t) \Delta t}{b-a}\right|^{3}\right) \Delta u-\frac{\left(\int_{a}^{b} f^{s}(t) \Delta t\right)^{3}}{(b-a)^{2}} \neq 0 .
$$

Then

$$
\frac{\mathfrak{M}_{r}^{r}(f)-\mathfrak{M}_{r}^{r}\left(\left|f^{s}-\mathfrak{M}_{s}^{s}(f)\right|^{\frac{1}{s}}\right)-\mathfrak{M}_{s}^{r}(f)}{\mathfrak{M}_{l}^{l}(f)-\mathfrak{M}_{l}^{l}\left(\left|f^{s}-\mathfrak{M}_{s}^{s}(f)\right|^{\frac{1}{s}}\right)-\mathfrak{M}_{s}^{l}(f)}=\frac{r(r-2 s)}{l(l-2 s)} \zeta^{r-l}
$$

holds for some $\zeta \in f\left([a, b)_{\mathbb{T}}\right)$, provided that the denominators in (4.4) are nonzero.

Proof. Equation (4.4) directly follows from Theorem 4.2 by taking $\alpha(x)=x^{r}$, $\beta(x)=x^{l}$ and $\gamma(x)=x^{s}$ in Theorem 4.2.

Remark 4.6. From Corollary 4.5, since $\zeta \in f\left([a, b)_{\mathbb{T}}\right)$, we obtain a new mean defined by

$$
\mathfrak{M}_{r, l}^{[s]}(f)=\left(\frac{l(l-2 s)}{r(r-2 s)} \frac{\mathfrak{M}_{r}^{r}(f)-\mathfrak{M}_{r}^{r}\left(\left|f^{s}-\mathfrak{M}_{s}^{s}(f)\right|^{\frac{1}{s}}\right)-\mathfrak{M}_{s}^{r}(f)}{\mathfrak{M}_{l}^{l}(f)-\mathfrak{M}_{l}^{l}\left(\left|f^{s}-\mathfrak{M}_{s}^{s}(f)\right|^{\frac{1}{s}}\right)-\mathfrak{M}_{s}^{l}(f)}\right)^{\frac{1}{r-l}}
$$

where $r, l, s>0, r \neq 2 s, l \neq 2 s$. We can extend these means to the limiting cases. To do so, let $r, l, s>0$. We define

$$
\begin{aligned}
& \mathfrak{M}_{l, l}^{[s]}(f)=\exp \left(\frac{P}{Q}-\frac{2(l-s)}{l(l-2 s)}\right), \quad l \neq 2 s, \\
& \mathfrak{M}_{l, 2 s}^{[s]}(f)=\mathfrak{M}_{2 s, l}^{[s]}(f)=\exp \left(\frac{2 s Q}{l(l-2 s) P_{1}}\right)^{\frac{1}{l-2 s}}, \quad l \neq 2 s, \\
& \mathfrak{M}_{2 s, 2 s}^{[s]}(f)=\exp \left(\frac{Q_{1}}{2 P_{1}}-\frac{1}{2 s}\right),
\end{aligned}
$$

where $P, Q, P_{1}$ and $Q_{1}$ are

$$
\begin{aligned}
P= & \frac{1}{b-a} \int_{a}^{b} f^{l}(t) \log f(t) \Delta t-\mathfrak{M}_{s}^{l}(f) \log \mathfrak{M}_{s}(f) \\
& -\frac{1}{s(b-a)} \int_{a}^{b}\left|f^{s}(t)-\mathfrak{M}_{s}^{s}(f)\right|^{\frac{l}{s}} \log \left|f^{s}(t)-\mathfrak{M}_{s}^{s}(f)\right| \Delta t \\
Q= & \mathfrak{M}_{l}^{l}(f)-\mathfrak{M}_{l}^{l}\left(\left|f^{s}-\mathfrak{M}_{s}^{s}(f)\right|^{\frac{1}{s}}\right)-\mathfrak{M}_{s}^{l}(f), \\
P_{1}= & \frac{1}{b-a} \int_{a}^{b} f^{2 s}(t) \log f(t) \Delta t-\mathfrak{M}_{s}^{2 s}(f) \log \mathfrak{M}_{s}(f) \\
& -\frac{1}{s(b-a)} \int_{a}^{b}\left|f^{s}(t)-\mathfrak{M}_{s}^{s}(f)\right|^{2} \log \left|f^{s}(t)-\mathfrak{M}_{s}^{s}(f)\right| \Delta t \\
Q_{1}= & \frac{1}{b-a} \int_{a}^{b} f^{2 s}(t)(\log f(t))^{2} \Delta t-\mathfrak{M}_{s}^{2 s}(f)\left(\log \mathfrak{M}_{s}(f)\right)^{2} \\
& -\frac{1}{s^{2}(b-a)} \int_{a}^{b}\left|f^{s}(t)-\mathfrak{M}_{s}^{s}(f)\right|^{2}\left(\log \left|f^{s}(t)-\mathfrak{M}_{s}^{s}(f)\right|\right)^{2} \Delta t
\end{aligned}
$$


Theorem 4.7. Let $a, b \in \mathbb{T}$ and $f \in \mathrm{C}_{\mathrm{rd}}\left([a, b)_{\mathbb{T}},[m, M]\right)$, where $0 \leq m<M<\infty$. Suppose $\alpha, \beta, \gamma \in \mathrm{C}^{2}([0, \infty), \mathbb{R})$ are strictly monotone such that

$\overline{\alpha \circ \gamma^{-1}}, \overline{\beta \circ \gamma^{-1}} \in \mathrm{C}^{1}((0, \infty), \mathbb{R}) \quad$ and $\quad\left(\alpha \circ \gamma^{-1}\right)(0)=\left(\beta \circ \gamma^{-1}\right)(0)=0$. If

$$
\begin{aligned}
& (b-a)\left((\gamma(m))^{3}+(\gamma(M))^{3}\right)-\int_{a}^{b}(\gamma \circ f)^{3}(t) \Delta t \\
& -(b-a)\left(\gamma(m)+\gamma(M)-\frac{1}{b-a} \int_{a}^{b}(\gamma \circ f)(t) \Delta t\right)^{3} \\
& -\frac{2}{\gamma(M)-\gamma(m)} \int_{a}^{b}\left[((\gamma \circ f)(t)-\gamma(m))(\gamma(M)-(\gamma \circ f)(t))^{3}\right. \\
& \left.+(\gamma(M)-(\gamma \circ f)(t))((\gamma \circ f)(t)-\gamma(m))^{3}\right] \Delta t \\
& -\int_{a}^{b}\left|(\gamma \circ f)(u)-\frac{1}{b-a} \int_{a}^{b}(\gamma \circ f)(t) \Delta t\right|^{3} \Delta u \neq 0
\end{aligned}
$$

then

$$
\begin{array}{r}
\frac{W_{\alpha}-X_{\alpha}-\frac{Y}{b-a} \int_{a}^{b}\left[\mathfrak{g}(t)\left(\alpha \circ \gamma^{-1}\right)(\mathfrak{h}(t))+\mathfrak{h}(t)\left(\alpha \circ \gamma^{-1}\right)(\mathfrak{g}(t))\right] \Delta t-Z_{\alpha}}{W_{\beta}-X_{\beta}-\frac{Y}{b-a} \int_{a}^{b}\left[\mathfrak{g}(t)\left(\beta \circ \gamma^{-1}\right)(\mathfrak{h}(t))+\mathfrak{h}(t)\left(\beta \circ \gamma^{-1}\right)(\mathfrak{g}(t))\right] \Delta t-Z_{\beta}} \\
=\frac{\gamma(\zeta)\left(\alpha^{\prime \prime}(\zeta) \gamma^{\prime}(\zeta)-\alpha^{\prime}(\zeta) \gamma^{\prime \prime}(\zeta)\right)-\alpha^{\prime}(\zeta)\left(\gamma^{\prime}(\zeta)\right)^{2}}{\gamma(\zeta)\left(\beta^{\prime \prime}(\zeta) \gamma^{\prime}(\zeta)-\beta^{\prime}(\zeta) \gamma^{\prime \prime}(\zeta)\right)-\beta^{\prime}(\zeta)\left(\gamma^{\prime}(\zeta)\right)^{2}}
\end{array}
$$

holds for some $\zeta \in f\left([a, b)_{\mathbb{T}}\right)$, provided that the denominators in (4.5) are nonzero, where

$$
\begin{gathered}
W_{\alpha}=\alpha(m)+\alpha(M)-\alpha\left(\mathfrak{M}_{\alpha}(f)\right), \\
X_{\alpha}=\left(\alpha \circ \gamma^{-1}\right)\left(\gamma(m)+\gamma(M)-\gamma\left(\mathfrak{M}_{\gamma}(f)\right)\right), \\
Z_{\alpha}=\alpha\left(\mathfrak{M}_{\alpha}\left(\gamma^{-1}\left(\left|(\gamma \circ f)-\gamma\left(\mathfrak{M}_{\gamma}(f)\right)\right|\right)\right)\right), \quad Y=\frac{2}{\gamma(M)-\gamma(m)}, \\
\mathfrak{g}=(\gamma \circ f)-\gamma(m), \quad \mathfrak{h}=\gamma(M)-(\gamma \circ f) .
\end{gathered}
$$

Proof. Replace the functions $f, \Psi$ and $\Phi$ in Theorem 3.5 by $\gamma \circ f, \alpha \circ \gamma^{-1}$ and $\beta \circ \gamma^{-1}$, respectively. The rest of the proof is analogous to the proof of Theorem 4.2 .

Remark 4.8. In Theorem 4.7, let

$$
\widetilde{\mathcal{F}}(\zeta)=\frac{\gamma(\zeta)\left(\alpha^{\prime \prime}(\zeta) \gamma^{\prime}(\zeta)-\alpha^{\prime}(\zeta) \gamma^{\prime \prime}(\zeta)\right)-\alpha^{\prime}(\zeta)\left(\gamma^{\prime}(\zeta)\right)^{2}}{\gamma(\zeta)\left(\beta^{\prime \prime}(\zeta) \gamma^{\prime}(\zeta)-\beta^{\prime}(\zeta) \gamma^{\prime \prime}(\zeta)\right)-\beta^{\prime}(\zeta)\left(\gamma^{\prime}(\zeta)\right)^{2}}
$$

and suppose $\widetilde{\mathcal{F}}$ is invertible. Then, since $\zeta$ is in the image of $f$, we obtain a new mean defined by

$$
\widetilde{\mathcal{F}}^{-1}\left(\frac{W_{\alpha}-X_{\alpha}-\frac{Y}{b-a} \int_{a}^{b}\left[\mathfrak{g}(t)\left(\alpha \circ \gamma^{-1}\right)(\mathfrak{h}(t))+\mathfrak{h}(t)\left(\alpha \circ \gamma^{-1}\right)(\mathfrak{g}(t))\right] \Delta t-Z_{\alpha}}{W_{\beta}-X_{\beta}-\frac{Y}{b-a} \int_{a}^{b}\left[\mathfrak{g}(t)\left(\beta \circ \gamma^{-1}\right)(\mathfrak{h}(t))+\mathfrak{h}(t)\left(\beta \circ \gamma^{-1}\right)(\mathfrak{g}(t))\right] \Delta t-Z_{\beta}}\right)
$$


Corollary 4.9. Let $a, b \in \mathbb{T}$ and $f \in \mathrm{C}_{\mathrm{rd}}\left([a, b)_{\mathbb{T}},[m, M]\right)$, where $0 \leq m<M<$ $\infty$. Suppose $r, l, s>0$ are such that $r \neq l, r \neq 2 s, l \neq 2 s$, and

$$
\begin{aligned}
& (b-a)\left(m^{3 s}+M^{3 s}\right)-\int_{a}^{b} f^{3 s}(t) \Delta t-(b-a)\left(m^{s}+M^{s}-\frac{1}{b-a} \int_{a}^{b} f^{s}(t) \Delta t\right)^{3} \\
& -\frac{2}{M^{s}-m^{s}} \int_{a}^{b}\left[\left(f^{s}(t)-m^{s}\right)\left(M^{s}-f^{s}(t)\right)^{3}\right. \\
& \left.+\left(M^{s}-f^{s}(t)\right)\left(f^{s}(t)-m^{s}\right)^{3}\right] \Delta t-\int_{a}^{b}\left|f^{s}(u)-\frac{1}{b-a} \int_{a}^{b} f^{s}(t) \Delta t\right|^{3} \Delta u \neq 0 .
\end{aligned}
$$

Then

$$
\frac{W_{r}-X_{r}-Y_{s}\left(\mathfrak{M}_{r}^{r}\left(\mathfrak{g}_{s}^{\frac{1}{r}} \mathfrak{h}_{s}^{\frac{1}{s}}\right)+\mathfrak{M}_{r}^{r}\left(\mathfrak{h}_{s}^{\frac{1}{r}} \mathfrak{g}_{s}^{\frac{1}{s}}\right)\right)-Z_{r}}{W_{l}-X_{l}-Y_{s}\left(\mathfrak{M}_{l}^{l}\left(\mathfrak{g}_{s}^{\frac{1}{l}} \mathfrak{h}_{s}^{\frac{1}{s}}\right)+\mathfrak{M}_{l}^{l}\left(\mathfrak{h}_{s}^{\frac{1}{l}} \mathfrak{g}_{s}^{\frac{1}{s}}\right)\right)-Z_{l}}=\frac{r(r-2 s)}{l(l-2 s)} \zeta^{r-l}
$$

holds for some $\zeta \in f\left([a, b)_{\mathbb{T}}\right)$, provided that the denominators in (4.6) are nonzero, where

$$
\begin{gathered}
W_{r}=m^{r}+M^{r}-\mathfrak{M}_{r}^{r}(f), \quad X_{r}=\left(m^{s}+M^{s}-\mathfrak{M}_{s}^{s}(f)\right)^{\frac{r}{s}} \\
Z_{r}=\mathfrak{M}_{r}^{r}\left(\left|f^{s}-\mathfrak{M}_{s}^{s}(f)\right|^{\frac{1}{s}}\right), \quad Y_{s}=\frac{2}{M^{s}-m^{s}} \\
\mathfrak{g}_{s}=f^{s}-m^{s}, \quad \mathfrak{h}_{s}=M^{s}-f^{s} .
\end{gathered}
$$

Remark 4.10. From Corollary 4.9, since $\zeta \in f\left([a, b)_{\mathbb{T}}\right)$, we obtain a new mean defined by

$$
\widetilde{\mathfrak{M}}_{r, l}^{[s]}(f)=\left(\frac{l(l-2 s)}{r(r-2 s)} \frac{W_{r}-X_{r}-Y_{s}\left(\mathfrak{M}_{r}^{r}\left(\mathfrak{g}_{s}^{\frac{1}{r}} \mathfrak{h}_{s}^{\frac{1}{s}}\right)+\mathfrak{M}_{r}^{r}\left(\mathfrak{h}_{s}^{\frac{1}{r}} \mathfrak{g}_{s}^{\frac{1}{s}}\right)\right)-Z_{r}}{W_{l}-X_{l}-Y_{s}\left(\mathfrak{M}_{l}^{l}\left(\mathfrak{g}_{s}^{\frac{1}{l}} \mathfrak{h}_{s}^{\frac{1}{s}}\right)+\mathfrak{M}_{l}^{l}\left(\mathfrak{h}_{s}^{\frac{1}{l}} \mathfrak{g}_{s}^{\frac{1}{s}}\right)\right)-Z_{l}}\right)^{\frac{1}{r-l}}
$$

where $r, l, s>0, r \neq 2 s, l \neq 2 s$. We can extend these means to the limiting cases. To do so, let $r, l, s>0$. We define

$$
\begin{aligned}
& \widetilde{\mathfrak{M}}_{l, l}^{[s]}(f)=\exp \left(\frac{\widetilde{P}}{\widetilde{Q}}-\frac{2(l-s)}{l(l-2 s)}\right), \quad l \neq 2 s, \\
& \widetilde{\mathfrak{M}}_{l, 2 s}^{[s]}(f)=\widetilde{\mathfrak{M}}_{2 s, l}^{[s]}(f)=\exp \left(\frac{2 s \widetilde{Q}}{l(l-2 s) \widetilde{P}_{1}}\right)^{\frac{1}{l-2 s}}, \quad l \neq 2 s, \\
& \widetilde{\mathfrak{M}}_{2 s, 2 s}^{[s]}(f)=\exp \left(\frac{\widetilde{Q}_{1}}{2 \widetilde{P}_{1}}-\frac{1}{2 s}\right),
\end{aligned}
$$

where $\widetilde{P}, \widetilde{Q}, \widetilde{P}_{1}$ and $\widetilde{Q}_{1}$ are defined by

$$
\begin{aligned}
\widetilde{P}= & m^{l} \log m+M^{l} \log M-\frac{1}{b-a} \int_{a}^{b} f^{l}(t) \log f(t) \Delta t \\
& -\frac{1}{s} X_{l} \log \left(m^{s}+M^{s}-\mathfrak{M}_{s}^{s}(f)\right)
\end{aligned}
$$


CAUCHY-TYPE MEANS FOR SUPERQUADRATIC FUNCTIONS

$$
\begin{aligned}
& -\frac{Y_{s}}{s(b-a)} \int_{a}^{b}\left[\mathfrak{g}_{s}(t) \mathfrak{h}_{s}^{\frac{l}{s}}(t) \log \left(\mathfrak{h}_{s}(t)\right)+\mathfrak{h}_{s}(t) \mathfrak{g}_{s}^{\frac{l}{s}}(t) \log \left(\mathfrak{g}_{s}(t)\right)\right] \Delta t \\
& -\frac{1}{s(b-a)} \int_{a}^{b}\left|f^{s}(t)-\mathfrak{M}_{s}^{s}(f)\right|^{\frac{l}{s}} \log \left|f^{s}(t)-\mathfrak{M}_{s}^{s}(f)\right| \Delta t \\
\widetilde{Q}= & W_{l}-X_{l}-Y_{s}\left(\mathfrak{M}_{l}^{l}\left(\mathfrak{g}_{s}^{\frac{1}{l}} \mathfrak{h}_{s}^{\frac{1}{s}}\right)+\mathfrak{M}_{l}^{l}\left(\mathfrak{h}_{s}^{\frac{1}{l}} \mathfrak{g}_{s}^{\frac{1}{s}}\right)\right)-Z_{l}, \\
\widetilde{P}_{1}= & m^{2 s} \log m+M^{2 s} \log M-\frac{1}{b-a} \int_{a}^{b} f^{2 s}(t) \log f(t) \Delta t \\
& -\frac{1}{s} X_{2 s} \log \left(m^{s}+M^{s}-\mathfrak{M}_{s}^{s}(f)\right) \\
& -\frac{Y_{s}}{s(b-a)} \int_{a}^{b}\left[\mathfrak{g}_{s}(t) \mathfrak{h}_{s}^{2}(t) \log \left(\mathfrak{h}_{s}(t)\right)+\mathfrak{h}_{s}(t) \mathfrak{g}_{s}^{2}(t) \log \left(\mathfrak{g}_{s}(t)\right)\right] \Delta t \\
& -\frac{1}{s(b-a)} \int_{a}^{b}\left|f^{s}(t)-\mathfrak{M}_{s}^{s}(f)\right|^{2} \log \left|f^{s}(t)-\mathfrak{M}_{s}^{s}(f)\right| \Delta t \\
\widetilde{Q}_{1}= & m^{2 s}(\log m)^{2}+M^{2 s}(\log M)^{2}-\frac{1}{b-a} \int_{a}^{b} f^{2 s}(t)(\log f(t))^{2} \Delta t \\
& -\frac{1}{s^{2}} X_{2 s}\left(\log \left(m^{s}+M^{s}-\mathfrak{M}_{s}^{s}(f)\right)\right)^{2} \\
& -\frac{Y_{s}}{s^{2}(b-a)} \int_{a}^{b}\left[\mathfrak{g}_{s}(t) \mathfrak{h}_{s}^{2}(t)\left(\log \left(\mathfrak{h}_{s}(t)\right)\right)^{2}+\mathfrak{h}_{s}(t) \mathfrak{g}_{s}^{2}(t)\left(\log \left(\mathfrak{g}_{s}(t)\right)\right)^{2}\right] \Delta t \\
& -\frac{1}{s^{2}(b-a)} \int_{a}^{b}\left|f^{s}(t)-\mathfrak{M}_{s}^{s}(f)\right|^{2}\left(\log \left|f^{s}(t)-\mathfrak{M}_{s}^{s}(f)\right|\right)^{2} \Delta t \\
& \\
& \\
&
\end{aligned}
$$

Theorem 4.11. Let $a, b \in \mathbb{T}$ and $f \in \mathrm{C}_{\mathrm{rd}}\left([a, b)_{\mathbb{T}},[m, M]\right)$, where $0 \leq m<M<$ $\infty$. Suppose $\alpha, \beta, \gamma \in \mathrm{C}^{2}([0, \infty), \mathbb{R})$ are strictly monotone such that

$$
\overline{\alpha \circ \gamma^{-1}}, \overline{\beta \circ \gamma^{-1}} \in \mathrm{C}^{1}((0, \infty), \mathbb{R}) \quad \text { and } \quad\left(\alpha \circ \gamma^{-1}\right)(0)=\left(\beta \circ \gamma^{-1}\right)(0)=0 \text {. }
$$

If

$$
\begin{aligned}
& \left.\int_{a}^{b}(\gamma \circ f)(t) \Delta t\left((\gamma(M))^{2}+(\gamma(m))^{2}+\gamma(M) \gamma(m)\right)\right) \\
& -(b-a) \gamma(M) \gamma(m)(\gamma(M)+\gamma(m))-\int_{a}^{b}(\gamma \circ f)^{3}(t) \Delta t \\
& -\frac{1}{\gamma(M)-\gamma(m)} \int_{a}^{b}\left[((\gamma \circ f)(t)-\gamma(m))(\gamma(M)-(\gamma \circ f)(t))^{3}\right. \\
& \left.+(\gamma(M)-(\gamma \circ f)(t))((\gamma \circ f)(t)-\gamma(m))^{3}\right] \Delta t \neq 0,
\end{aligned}
$$

then

$$
\begin{array}{r}
\frac{(b-a) E_{\alpha}-\int_{a}^{b}\left[\mathfrak{g}(t)\left(\alpha \circ \gamma^{-1}\right)(\mathfrak{h}(t))+\mathfrak{h}(t)\left(\alpha \circ \gamma^{-1}\right)(\mathfrak{g}(t))\right] \Delta t-(b-a) F_{\alpha}}{(b-a) E_{\beta}-\int_{a}^{b}\left[\mathfrak{g}(t)\left(\beta \circ \gamma^{-1}\right)(\mathfrak{h}(t))+\mathfrak{h}(t)\left(\beta \circ \gamma^{-1}\right)(\mathfrak{g}(t))\right] \Delta t-(b-a) F_{\beta}} \\
=\frac{\gamma(\zeta)\left(\alpha^{\prime \prime}(\zeta) \gamma^{\prime}(\zeta)-\alpha^{\prime}(\zeta) \gamma^{\prime \prime}(\zeta)\right)-\alpha^{\prime}(\zeta)\left(\gamma^{\prime}(\zeta)\right)^{2}}{\gamma(\zeta)\left(\beta^{\prime \prime}(\zeta) \gamma^{\prime}(\zeta)-\beta^{\prime}(\zeta) \gamma^{\prime \prime}(\zeta)\right)-\beta^{\prime}(\zeta)\left(\gamma^{\prime}(\zeta)\right)^{2}}
\end{array}
$$


holds for some $\zeta \in f\left([a, b)_{\mathbb{T}}\right)$, provided that the denominators in (4.7) are nonzero, where $\mathfrak{g}$ and $\mathfrak{h}$ are defined as in Theorem 4.7 and

$$
\begin{gathered}
E_{\alpha}=\left(\gamma(M)-\gamma\left(\mathfrak{M}_{\gamma}(f)\right)\right) \alpha(m)+\left(\gamma\left(\mathfrak{M}_{\gamma}(f)\right)-\gamma(m)\right) \alpha(M), \\
F_{\alpha}=(\gamma(M)-\gamma(m)) \alpha\left(\mathfrak{M}_{\alpha}(f)\right) .
\end{gathered}
$$

Proof. Replace the functions $f, \Psi$ and $\Phi$ in Theorem 3.5 by $\gamma \circ f, \alpha \circ \gamma^{-1}$ and $\beta \circ \gamma^{-1}$, respectively. The rest of the proof is analogous to the proof of Theorem 4.2 .

Remark 4.12. In Theorem 4.11, let

$$
\widehat{\mathcal{F}}(\zeta)=\frac{\gamma(\zeta)\left(\alpha^{\prime \prime}(\zeta) \gamma^{\prime}(\zeta)-\alpha^{\prime}(\zeta) \gamma^{\prime \prime}(\zeta)\right)-\alpha^{\prime}(\zeta)\left(\gamma^{\prime}(\zeta)\right)^{2}}{\gamma(\zeta)\left(\beta^{\prime \prime}(\zeta) \gamma^{\prime}(\zeta)-\beta^{\prime}(\zeta) \gamma^{\prime \prime}(\zeta)\right)-\beta^{\prime}(\zeta)\left(\gamma^{\prime}(\zeta)\right)^{2}}
$$

and suppose $\widehat{\mathcal{F}}$ is invertible. Then, since $\zeta$ is in the image of $f$, we obtain a new mean defined by

$\widehat{\mathcal{F}}^{-1}\left(\frac{(b-a) E_{\alpha}-\int_{a}^{b}\left[\mathfrak{g}(t)\left(\alpha \circ \gamma^{-1}\right)(\mathfrak{h}(t))+\mathfrak{h}(t)\left(\alpha \circ \gamma^{-1}\right)(\mathfrak{g}(t))\right] \Delta t-(b-a) F_{\alpha}}{(b-a) E_{\beta}-\int_{a}^{b}\left[\mathfrak{g}(t)\left(\beta \circ \gamma^{-1}\right)(\mathfrak{h}(t))+\mathfrak{h}(t)\left(\beta \circ \gamma^{-1}\right)(\mathfrak{g}(t))\right] \Delta t-(b-a) F_{\beta}}\right)$.

Corollary 4.13. Let $a, b \in \mathbb{T}$ and $f \in \mathrm{C}_{\mathrm{rd}}\left([a, b)_{\mathbb{T}},[m, M]\right)$, where $0 \leq m<M<$ $\infty$. Suppose $r, l, s>0$ are such that $r \neq l, r \neq 2 s, l \neq 2 s$, and

$$
\begin{aligned}
& \int_{a}^{b} f^{s}(t) \Delta t\left(M^{2 s}+m^{2 s}+(M m)^{s}\right)-(b-a)(M m)^{s}\left(M^{s}+m^{s}\right)-\int_{a}^{b} f^{3 s}(t) \Delta t \\
& -\frac{\int_{a}^{b}\left[\left(f^{s}(t)-m^{s}\right)\left(M^{s}-f^{s}(t)\right)^{3}+\left(M^{s}-f^{s}(t)\right)\left(f^{s}(t)-m^{s}\right)^{3}\right] \Delta t}{M^{s}-m^{s}} \neq 0 .
\end{aligned}
$$

Then

$$
\frac{E_{r}-\mathfrak{M}_{r}^{r}\left(\mathfrak{g}_{s}^{\frac{1}{r}} \mathfrak{h}_{s}^{\frac{1}{s}}\right)-\mathfrak{M}_{r}^{r}\left(\mathfrak{h}_{s}^{\frac{1}{r}} \mathfrak{g}_{s}^{\frac{1}{s}}\right)-F_{r}}{E_{l}-\mathfrak{M}_{l}^{l}\left(\mathfrak{g}_{s}^{\frac{1}{l}} \mathfrak{h}_{s}^{\frac{1}{s}}\right)-\mathfrak{M}_{l}^{l}\left(\mathfrak{h}_{s}^{\frac{1}{l}} \mathfrak{g}_{s}^{\frac{1}{s}}\right)-F_{l}}=\frac{r(r-2 s)}{l(l-2 s)} \zeta^{r-l}
$$

holds for some $\zeta \in f\left([a, b)_{\mathbb{T}}\right)$, provided that the denominators in (4.8) are nonzero, where $\mathfrak{g}_{s}$ and $\mathfrak{h}_{s}$ are defined as in Corollary 4.9 and

$$
E_{r}=\left(M^{s}-\mathfrak{M}_{s}^{s}(f)\right) m^{r}+\left(\mathfrak{M}_{s}^{s}(f)-m^{s}\right) M^{r}, \quad F_{r}=\left(M^{s}-m^{s}\right) \mathfrak{M}_{r}^{r}(f) .
$$

Remark 4.14. From Corollary 4.13, since $\zeta \in f\left([a, b)_{\mathbb{T}}\right)$, we obtain a new mean defined by

$$
\widehat{\mathfrak{M}}_{r, l}^{[s]}(f)=\left(\frac{l(l-2 s)}{r(r-2 s)} \frac{E_{r}-\mathfrak{M}_{r}^{r}\left(\mathfrak{g}_{s}^{\frac{1}{r}} \mathfrak{h}_{s}^{\frac{1}{s}}\right)-\mathfrak{M}_{r}^{r}\left(\mathfrak{h}_{s}^{\frac{1}{r}} \mathfrak{g}_{s}^{\frac{1}{s}}\right)-F_{r}}{E_{l}-\mathfrak{M}_{l}^{l}\left(\mathfrak{g}_{s}^{\frac{1}{l}} \mathfrak{h}_{s}^{\frac{1}{s}}\right)-\mathfrak{M}_{l}^{l}\left(\mathfrak{h}_{s}^{\frac{1}{l}} \mathfrak{g}_{s}^{\frac{1}{s}}\right)-F_{l}}\right)^{\frac{1}{r-l}}
$$

where $r, l, s>0, r \neq 2 s, l \neq 2 s$. We can extend these means to the limiting cases. To do so, let $r, l, s>0$. We define

$$
\widehat{\mathfrak{M}}_{l, l}^{[s]}(f)=\exp \left(\frac{\widehat{P}}{\widehat{Q}}-\frac{2(l-s)}{l(l-2 s)}\right), \quad l \neq 2 s
$$




$$
\begin{aligned}
& \widehat{\mathfrak{M}}_{l, 2 s}^{[s]}(f)=\widehat{\mathfrak{M}}_{2 s, l}^{[s]}(f)=\exp \left(\frac{2 s \widehat{Q}}{l(l-2 s) \widehat{P}_{1}}\right)^{\frac{1}{l-2 s}}, \quad l \neq 2 s, \\
& \widehat{\mathfrak{M}}_{2 s, 2 s}^{[s]}(f)=\exp \left(\frac{\widehat{Q}_{1}}{2 \widehat{P}_{1}}-\frac{1}{2 s}\right),
\end{aligned}
$$

where $\widehat{P}, \widehat{Q}, \widehat{P}_{1}$ and $\widehat{Q}_{1}$ are defined by

$$
\begin{aligned}
\widehat{P}= & \left(M^{s}-\mathfrak{M}_{s}^{s}(f)\right) m^{l} \log m+\left(\mathfrak{M}_{s}^{s}(f)-m^{s}\right) M^{l} \log M \\
& -\frac{1}{s(b-a)} \int_{a}^{b}\left[\mathfrak{g}_{s}(t) \mathfrak{h}_{s}^{\frac{l}{s}}(t) \log \left(\mathfrak{h}_{s}(t)\right)+\mathfrak{h}_{s}(t) \mathfrak{g}_{s}^{\frac{l}{s}}(t) \log \left(\mathfrak{g}_{s}(t)\right)\right] \Delta t \\
& -\frac{M^{s}-m^{s}}{b-a} \int_{a}^{b} f^{l}(t) \log f(t) \Delta t \\
\widehat{Q}= & E_{l}-\mathfrak{M}_{l}^{l}\left(\mathfrak{g}_{s}^{\frac{1}{l}} \mathfrak{h}_{s}^{\frac{1}{s}}\right)-\mathfrak{M}_{l}^{l}\left(\mathfrak{h}_{s}^{\frac{1}{l}} \mathfrak{g}_{s}^{\frac{1}{s}}\right)-F_{l}, \\
\widehat{P}_{1}= & \left(M^{s}-\mathfrak{M}_{s}^{s}(f)\right) m^{2 s} \log m+\left(\mathfrak{M}_{s}^{s}(f)-m^{s}\right) M^{2 s} \log M \\
& -\frac{1}{s(b-a)} \int_{a}^{b}\left[\mathfrak{g}_{s}(t) \mathfrak{h}_{s}^{2}(t) \log \left(\mathfrak{h}_{s}(t)\right)+\mathfrak{h}_{s}(t) \mathfrak{g}_{s}^{2}(t) \log \left(\mathfrak{g}_{s}(t)\right)\right] \Delta t \\
& -\frac{M^{s}-m^{s}}{b-a} \int_{a}^{b} f^{2 s}(t) \log f(t) \Delta t, \\
\widehat{Q}_{1}= & \left(M^{s}-\mathfrak{M}_{s}^{s}(f)\right) m^{2 s}(\log m)^{2}+\left(\mathfrak{M}_{s}^{s}(f)-m^{s}\right) M^{2 s}(\log M)^{2} \\
& -\frac{1}{s^{2}(b-a)} \int_{a}^{b}\left[\mathfrak{g}_{s}(t) \mathfrak{h}_{s}^{2}(t)\left(\log \left(\mathfrak{h}_{s}(t)\right)\right)^{2}+\mathfrak{h}_{s}(t) \mathfrak{g}_{s}^{2}(t)\left(\log \left(\mathfrak{g}_{s}(t)\right)\right)^{2}\right] \Delta t \\
& -\frac{M^{s}-m^{s}}{b-a} \int_{a}^{b} f^{2 s}(t)(\log f(t))^{2} \Delta t
\end{aligned}
$$

\section{Exponential Convexity and Logarithmic Convexity}

Applying the functional $\mathcal{J}_{\Psi}$ to the function $\Psi_{s}$ defined in Lemma 1.8, we obtain

$$
\begin{aligned}
\mathcal{J}_{\Psi_{s}}= & \frac{1}{s(s-2)}\left\{\int_{a}^{b}\left[f^{s}(u)-\left|f(u)-\frac{\int_{a}^{b} f(t) \Delta t}{b-a}\right|^{s}\right] \Delta u\right. \\
& \left.-(b-a)\left(\frac{\int_{a}^{b} f(t) \Delta t}{b-a}\right)^{s}\right\}, \quad s \neq 2
\end{aligned}
$$

and

$$
\begin{aligned}
\mathcal{J}_{\Psi_{2}}= & \frac{1}{2}\left\{\int _ { a } ^ { b } \left[f^{2}(u) \log f(u)\right.\right. \\
& \left.-\left|f(u)-\frac{\int_{a}^{b} f(t) \Delta t}{b-a}\right|^{2} \log \left|f(u)-\frac{\int_{a}^{b} f(t) \Delta t}{b-a}\right|\right] \Delta u
\end{aligned}
$$




$$
\left.-\frac{1}{b-a}\left(\int_{a}^{b} f(t) \Delta t\right)^{2} \log \left(\frac{\int_{a}^{b} f(t) \Delta t}{b-a}\right)\right\} \text {. }
$$

Theorem 5.1. Let $\mathcal{J}_{\Psi_{s}}$ be defined as in (5.1)-(5.2). Then

(i) for all $n \in \mathbb{N}$ and for all $p_{i}>0, p_{i j}=\frac{p_{i}+p_{j}}{2}, 1 \leq i, j \leq n$, the matrix $\left[\mathcal{J}_{\Psi_{p_{i j}}}\right]_{i, j=1}^{n}$ is positive semidefinite;

(ii) the function $s \mapsto \mathcal{J}_{\Psi_{s}}$ is exponentially convex;

(iii) if $\mathcal{J}_{\Psi_{s}}>0$, then the function $s \mapsto \mathcal{J}_{\Psi_{s}}$ is $\log$-convex, i.e., for $0<r<s<$ $w$, we have

$$
\left(\mathcal{J}_{\Psi_{s}}\right)^{w-r} \leq\left(\mathcal{J}_{\Psi_{r}}\right)^{w-s}\left(\mathcal{J}_{\Psi_{w}}\right)^{s-r}
$$

Proof. To show (i), let

$$
\Lambda(x)=\sum_{i, j=1}^{n} v_{i} v_{j} \Psi_{p_{i j}}(x)
$$

Then

$$
\bar{\Lambda}^{\prime}(x)=\sum_{i, j=1}^{n} v_{i} v_{j} x^{\frac{p_{i j}}{2}-3}=\left(\sum_{i=1}^{n} v_{i} x^{\frac{p_{i}-3}{2}}\right)^{2} \geq 0
$$

and $\Lambda(0)=0$. Thus $\Lambda$ is superquadratic. Now using $\Lambda$ instead of $\Psi$ in (3.1), we obtain

$$
\mathcal{J}_{\Lambda}=\sum_{i, j=1}^{n} v_{i} v_{j} \mathcal{J}_{\Psi_{p_{i j}}} \geq 0
$$

Hence the matrix $\left[\mathcal{J}_{\Psi_{p_{i j}}}\right]_{i, j=1}^{n}$ is positive semidefinite.

Now we show (ii). Because $\lim _{s \rightarrow 2} \mathcal{J}_{\Psi_{s}}=\mathcal{J}_{\Psi_{2}}$, the function $s \mapsto \mathcal{J}_{\Psi_{s}}$ is continuous on $\mathbb{R}_{+}$. Hence by (5.3) and Proposition 1.11, the function $s \mapsto \mathcal{J}_{\Psi_{s}}$ is exponentially convex.

Finally, we show (iii). Because the function $s \mapsto \mathcal{J}_{\Psi_{s}}$ is exponentially convex, if $\mathcal{J}_{\Psi_{s}}>0$, then by Remark 1.12, the function $s \mapsto \mathcal{J}_{\Psi_{s}}$ is log-convex. 
Corollary 5.2. Let $a, b \in \mathbb{T}$ and $f \in \mathrm{C}_{\mathrm{rd}}\left([a, b)_{\mathbb{T}}, I\right)$ be positive and define

$$
\mathcal{D}_{s}= \begin{cases}\int_{a}^{b}\left[f^{s}(u)-\left|f(u)-\frac{\int_{a}^{b} f(t) \Delta t}{b-a}\right|^{s}\right] \Delta u & s \neq 2 \\ -(b-a)\left(\frac{\int_{a}^{b} f(t) \Delta t}{b-a}\right)^{s}, & \\ \int_{a}^{b}\left[f^{2}(u) \log f(u)-\left|f(u)-\frac{\int_{a}^{b} f(t) \Delta t}{b-a}\right|^{2}\right. \\ \log \left[f(u)-\frac{\int_{a}^{b} f(t) \Delta t}{b-a} \mid\right] \Delta u \\ -\frac{1}{b-a}\left(\int_{a}^{b} f(t) \Delta t\right)^{2} \log \left(\frac{\int_{a}^{b} f(t) \Delta t}{b-a}\right), \quad s=2 .\end{cases}
$$

Then

(i) for $s>4$,

$$
\begin{aligned}
\frac{\int_{a}^{b} f^{s}(t) \Delta t}{b-a} \geq\left(\frac{\int_{a}^{b} f(t) \Delta t}{b-a}\right)^{s}+\frac{1}{b-a} \int_{a}^{b}\left|f(u)-\frac{\int_{a}^{b} f(t) \Delta t}{b-a}\right|^{s} \Delta u & +\frac{s(s-2)}{3(b-a)}\left(\frac{3 \mathcal{D}_{4}}{8 \mathcal{D}_{3}}\right)^{s-3} \mathcal{D}_{3} ;
\end{aligned}
$$

(ii) for $1<s<2$,

$$
\begin{aligned}
& \frac{\int_{a}^{b} f^{s}(t) \Delta t}{b-a} \leq\left(\frac{\int_{a}^{b} f(t) \Delta t}{b-a}\right)^{s}+\frac{1}{b-a} \int_{a}^{b}\left|f(u)-\frac{\int_{a}^{b} f(t) \Delta t}{b-a}\right|^{s} \Delta u \\
& \quad+\frac{s(s-2)}{b-a}\left(\frac{\mathcal{D}_{2}}{2 \int_{a}^{b}\left|f(u)-\frac{\int_{a}^{b} f(t) \Delta t}{b-a}\right| \Delta u}\right) \int_{a}^{b-1}\left|f(u)-\frac{\int_{a}^{b} f(t) \Delta t}{b-a}\right| \Delta u
\end{aligned}
$$

(iii) for $2<s<3$,

$$
\begin{aligned}
\frac{\int_{a}^{b} f^{s}(t) \Delta t}{b-a} \leq\left(\frac{\int_{a}^{b} f(t) \Delta t}{b-a}\right)^{s}+\frac{1}{b-a} \int_{a}^{b}\left|f(u)-\frac{\int_{a}^{b} f(t) \Delta t}{b-a}\right|^{s} \Delta u & +\frac{s(s-2)}{2(b-a)}\left(\frac{2 \mathcal{D}_{3}}{3 \mathcal{D}_{2}}\right)^{s-2} \mathcal{D}_{2} ;
\end{aligned}
$$

(iv) for $3<s<4$,

$\frac{\int_{a}^{b} f^{s}(t) \Delta t}{b-a} \leq\left(\frac{\int_{a}^{b} f(t) \Delta t}{b-a}\right)^{s}+\frac{1}{b-a} \int_{a}^{b}\left|f(u)-\frac{\int_{a}^{b} f(t) \Delta t}{b-a}\right|^{s} \Delta u$ 


$$
+\frac{s(s-2)}{3(b-a)}\left(\frac{3 \mathcal{D}_{4}}{8 \mathcal{D}_{3}}\right)^{s-3} \mathcal{D}_{3} .
$$

Proof. The results follow from Theorem 5.1 (iii).

Example 5.3 (See [2]). Let us consider the discrete form of $\mathcal{D}_{s}$. For this, let $[a, b)=\{1,2\}, f(1)=x, f(2)=y$ such that $y \geq x \geq 0$. Then $\mathcal{D}_{s}$ becomes

$$
\mathcal{D}_{s}=d_{s}=x^{s}+y^{s}-2\left(\frac{x+y}{2}\right)^{s}-2\left(\frac{y-x}{2}\right)^{s} .
$$

For $s>4$, we obtain the inequality

$$
\begin{aligned}
d_{s} & \geq \frac{s(s-2)}{3}\left(\frac{3 d_{4}}{8 d_{3}}\right)^{s-3} d_{3} \\
& =\frac{s(s-2)}{3}\left(\frac{3^{2}(y+x)^{2}}{4^{2}(y+2 x)}\right)^{s-3} \frac{(y-x)^{2}(y+2 x)}{2} .
\end{aligned}
$$

If $3<s<4$, we have

$$
d_{s} \leq \frac{s(s-2)}{3}\left(\frac{3^{2}(y+x)^{2}}{4^{2}(y+2 x)}\right)^{s-3} \frac{(y-x)^{2}(y+2 x)}{2} .
$$

Therefore for $s=1$, the inequality becomes

$$
-(y-x) \leq-\frac{1}{3 \cdot 2}\left(\frac{4^{2}}{3^{2}}\right)^{2} \frac{(y+2 x)^{3}(y-x)^{2}}{(y+x)^{4}} .
$$

Theorem 5.4. Suppose $p, q \in \mathbb{R}$ are such that $1<p<2$ and $\frac{1}{p}+\frac{1}{q}=1$. Let $a, b \in \mathbb{T}$ and $f, g \in \mathrm{C}_{\mathrm{rd}}\left([a, b)_{\mathbb{T}},[0, \infty)\right)$ be such that $\int_{a}^{b} g^{q}(t) \Delta t>0$. Then

$$
\begin{aligned}
& \frac{1}{p(p-2)}\left(\left(\left(\int_{a}^{b} f^{p}(t) \Delta t-\int_{a}^{b} g(u) h^{p}(u) \Delta u\right)^{\frac{1}{p}}\left(\int_{a}^{b} g^{q}(t) \Delta t\right)^{\frac{1}{q}}\right)^{p}\right. \\
& \left.\quad-\left(\int_{a}^{b} f(t) g(t) \Delta t\right)^{p}\right) \\
& \leq \frac{1}{2^{p-1}}\left(\int_{a}^{b} g(u) h(u) \Delta u\right)^{2-p} \\
& \left(\int _ { a } ^ { b } g ^ { q } ( t ) \Delta t \left(\int_{a}^{b} f^{2}(t) g^{2-q}(t) \log \left(f(t) g^{1-q}(t)\right) \Delta t\right.\right. \\
& \left.-\int_{a}^{b} g^{2-q}(u) h^{2}(u) \log \left(g^{1-q}(u) h(u)\right) \Delta u\right) \\
& \left.-\left(\int_{a}^{b} f(t) g(t) \Delta t\right)^{2} \log \left(\frac{\int_{a}^{b} f(t) g(t) \Delta t}{\int_{a}^{b} g^{q}(t) \Delta t}\right)\right)^{p-1}
\end{aligned}
$$


holds, where

$$
h(u)=\left|f(u)-g^{q-1}(u) \frac{\int_{a}^{b} f(t) g(t) \Delta t}{\int_{a}^{b} g^{q}(t) \Delta t}\right| .
$$

Proof. In Theorem 5.1 (iii), let $r=1, s=p, w=2$, so that $1<p<2$. Then we have

$$
\left(\mathcal{J}_{\Psi_{p}}\right)^{1} \leq\left(\mathcal{J}_{\Phi_{1}}\right)^{2-p}\left(\mathcal{J}_{\Phi_{2}}\right)^{p-1} .
$$

By replacing $\frac{\int_{a}^{b} f(t) \Delta t}{b-a}$ with $\frac{\int_{a}^{b} k(t) f(t) \Delta t}{\int_{a}^{b} k(t) \Delta t}$, where $k \in \mathrm{C}_{\mathrm{rd}}\left([a, b)_{\mathbb{T}},[0, \infty)\right)$ is such that $\int_{a}^{b} k(t) \Delta t>0$, we get

$$
\begin{aligned}
\frac{1}{p(p-2)} & \left(\int_{a}^{b} k(t) f^{p}(t) \Delta t-\int_{a}^{b} k(u)\left|f(u)-\frac{\int_{a}^{b} k(t) f(t) \Delta t}{\int_{a}^{b} k(t) \Delta t}\right|^{p} \Delta u\right. \\
& \left.-\int_{a}^{b} k(t) \Delta t\left(\frac{\int_{a}^{b} k(t) f(t) \Delta t}{\int_{a}^{b} k(t) \Delta t}\right)^{p}\right) \\
\leq \frac{1}{2^{p-1}}\{ & \left(\int_{a}^{b} k(t)\left|f(u)-\frac{\int_{a}^{b} k(t) f(t) \Delta t}{\int_{a}^{b} k(t) \Delta t}\right| \Delta u\right)^{2-p}\left(\int_{a}^{b} k(t) f^{2}(t) \log f(t) \Delta t\right. \\
& -\int_{a}^{b} k(u)\left|f(u)-\frac{\int_{a}^{b} k(t) f(t) \Delta t}{\int_{a}^{b} k(t) \Delta t}\right|^{2} \log \left|f(u)-\frac{\int_{a}^{b} k(t) f(t) \Delta t}{\int_{a}^{b} k(t) \Delta t}\right| \Delta u \\
& \left.\left.-\frac{1}{\int_{a}^{b} k(t) \Delta t}\left(\int_{a}^{b} k(t) f(t) \Delta t\right)^{2} \log \left(\frac{\int_{a}^{b} k(t) f(t) \Delta t}{\int_{a}^{b} k(t) \Delta t}\right)\right)^{p-1}\right\} .
\end{aligned}
$$

Now replacing $k$ by $g^{q}$ and $f$ by $f g^{1-q}$, after some calculation, we get the required result.

Remark 5.5. Theorem 5.4 refines the time scales Hölder inequality for superquadratic functions as given in [6].

Theorem 5.6. Let $\mathcal{J}_{\Psi_{s}}$ and $\mathcal{J}_{\Psi_{2}}$ be positive. Then for $r, l, v, w>0$ such that $r \leq v, l \leq w$, we have

$$
\mathfrak{M}_{r, l}^{[s]}(f) \leq \mathfrak{M}_{v, w}^{[s]}(f)
$$

Proof. Since $\mathcal{J}_{\Psi_{s}}$ is positive, by Theorem 5.1, $\mathcal{J}_{\Psi_{s}}$ is log-convex. Now by using Lemma 1.13, for $r, l, v, w>0$ such that $r \leq v, l \leq w, r \neq l, v \neq w$, we have

$$
\left(\frac{\mathcal{J}_{\Psi_{r}}}{\mathcal{J}_{\Psi_{l}}}\right)^{\frac{1}{r-l}} \leq\left(\frac{\mathcal{J}_{\Psi_{v}}}{\mathcal{J}_{\Psi_{w}}}\right)^{\frac{1}{v-w}}
$$

By substituting $\frac{r}{s}$ for $r, \frac{l}{s}$ for $l, \frac{u}{s}$ for $u, \frac{v}{s}$ for $v, f^{s}$ for $f$ and from the continuity of $\mathcal{J}_{\Psi_{s}}$, we obtain our required result.

Theorem 5.7. Theorem 5.1 is still valid if we replace $\Psi_{s}$ by $\varphi_{s}$ as defined in Lemma 1.9. 
Proof. As in the proof of Theorem 5.1, consider

$$
\Omega(x)=\sum_{i, j=1}^{n} v_{i} v_{j} \varphi_{p_{i j}}(x) .
$$

Then

$$
\bar{\Omega}^{\prime}(x)=\left(\sum_{i=1}^{n} v_{i} e^{\frac{p_{i}}{2} x}\right)^{2} \geq 0
$$

and $\Omega(0)=0$. Thus $\Omega$ is superquadratic. Now using $\Omega$ instead of $\Psi$ in (3.1), we obtain our required result.

Corollary 5.8. Let $a, b \in \mathbb{T}$ and $f \in \mathrm{C}_{\mathrm{rd}}\left([a, b)_{\mathbb{T}}, I\right)$ be positive. Let $r, s \in \mathbb{R}$, $r \neq s$. Then we have

$$
\begin{aligned}
& \mathfrak{M}_{r, s}(f) \\
= & \left(\frac{s^{3}\left(r \int_{a}^{b} f^{r}(t) \log f(t) \Delta t-A_{r}-r \int_{a}^{b} \mathcal{B}(t) e^{r \mathcal{B}(t)} \Delta t+\int_{a}^{b} e^{r \mathcal{B}(t)} \Delta t-1\right)}{r^{3}\left(s \int_{a}^{b} f^{s}(t) \log f(t) \Delta t-A_{s}-s \int_{a}^{b} \mathcal{B}(t) e^{s \mathcal{B}(t)} \Delta t+\int_{a}^{b} e^{s \mathcal{B}(t)} \Delta t-1\right)}\right)^{\frac{1}{r-s}},
\end{aligned}
$$

provided that the occurring denominators are nonzero, where

$$
A_{r}=(b-a)\left(\mathfrak{M}_{r}^{r}(f)+\mathfrak{M}_{0}^{r}(f) \log \left(\mathfrak{M}_{0}^{r}(f)\right)-\mathfrak{M}_{0}^{r}(f)\right), \quad \mathcal{B}(t)=\left|\log \left(\frac{f(t)}{\mathfrak{M}_{0}(f)}\right)\right|
$$

Proof. The proof follows from Theorem 3.2 by replacing $\Psi, \Phi$ and $f$ with $\varphi_{r}, \varphi_{s}$ and $\log f$, respectively.

Remark 5.9. For the limiting cases of Cauchy-type means defined in Corollary 5.8 , we have

$$
\mathfrak{M}_{s, s}(f)=\exp \left(\frac{B}{C}-\frac{3}{s}\right), \quad s \neq 0 \quad \text { and } \quad \mathfrak{M}_{0,0}(f)=\exp \left(\frac{3 B_{1}}{8 C_{1}}\right),
$$

where

$$
\begin{aligned}
B= & s\left(\int_{a}^{b} f^{s}(t)(\log f(t))^{2} \Delta t-(b-a) \mathfrak{M}_{0}^{s}(f)\left(\log \left(\mathfrak{M}_{0}(f)\right)\right)^{2}\right. \\
& \left.-\int_{a}^{b} \mathcal{B}^{2}(t) e^{s \mathcal{B}(t)} \Delta t\right) \\
C= & s \int_{a}^{b} f^{s}(t) \log f(t) \Delta t-(b-a) A_{s}-s \int_{a}^{b} \mathcal{B}(t) e^{s \mathcal{B}(t)} \Delta t+\int_{a}^{b} e^{s \mathcal{B}(t)} \Delta t-1, \\
B_{1}= & \int_{a}^{b}(\log f(t))^{4} \Delta t-(b-a)\left(\log \left(\mathfrak{M}_{0}(f)\right)\right)^{4}-\int_{a}^{b} \mathcal{B}^{4}(t) \Delta t \\
C_{1}= & \int_{a}^{b}(\log f(t))^{3} \Delta t-(b-a)\left(\log \left(\mathfrak{M}_{0}(f)\right)\right)^{3}-\int_{a}^{b} \mathcal{B}^{3}(t) \Delta t .
\end{aligned}
$$

Theorem 5.10. Let $\mathcal{J}_{\Psi_{s}}$ be positive. Then for $r, l, v, w>0$ such that $r \leq v$, $l \leq w$, we have

$$
\mathfrak{M}_{r, l}(f) \leq \mathfrak{M}_{v, w}(f)
$$


Proof. See the proof of Theorem 5.6.

We can obtain corresponding results for $\widetilde{\mathcal{J}}_{\Psi_{s}}$ and $\widehat{\mathcal{J}}_{\Psi_{s}}$ analogously as in the case of $\mathcal{J}_{\Psi_{s}}$.

Theorem 5.11. (i) For all $n \in \mathbb{N}$ and for all $p_{i}>0, p_{i j}=\frac{p_{i}+p_{j}}{2}, 1 \leq$ $i, j \leq n$, the matrix $\left[\widetilde{\mathcal{J}}_{\Psi_{p_{i j}}}\right]_{i, j=1}^{n}$ is positive semidefinite;

(ii) the function $s \mapsto \widetilde{\mathcal{J}}_{\Psi_{s}}$ is exponentially convex;

(iii) if $\widetilde{\mathcal{J}}_{\Psi_{s}}>0$, then the function $s \mapsto \widetilde{\mathcal{J}}_{\Psi_{s}}$ is log-convex, i.e., for $0<r<s<$ $w$, we have

$$
\widetilde{\mathcal{J}}_{\Psi_{s}}^{w-r} \leq \widetilde{\mathcal{J}}_{\Psi_{r}}^{w-s} \widetilde{\mathcal{J}}_{\Psi_{w}}^{s-r}
$$

Corollary 5.12. Let $a, b \in \mathbb{T}$ and $f \in \mathrm{C}_{\mathrm{rd}}\left([a, b)_{\mathbb{T}},[m, M]\right)$, where $0 \leq m<M<$ $\infty$. Suppose

$$
\widetilde{\mathcal{D}}_{s}= \begin{cases}(b-a)\left(m^{s}+M^{s}\right)-\int_{a}^{b} f^{s}(t) \Delta t & \\ -(b-a)\left(m+M-\frac{1}{b-a} \int_{a}^{b} f(t) \Delta t\right)^{s}-K_{s}, & s \neq 2 \\ (b-a)\left(m^{2} \log m+M^{2} \log M\right)-\int_{a}^{b} f^{2}(t) \log f(t) \Delta t & \\ -(b-a)\left(m+M-\frac{1}{b-a} \int_{a}^{b} f(t) \Delta t\right)^{2} & s=2, \\ \log \left(m+M-\frac{1}{b-a} \int_{a}^{b} f(t) \Delta t\right)-K_{2}, & s=2\end{cases}
$$

where

$$
\begin{aligned}
K_{s}= & \frac{2}{M-m} \int_{a}^{b}\left[(f(t)-m)(M-f(t))^{s}+(M-f(t))(f(t)-m)^{s}\right] \Delta t \\
& +\int_{a}^{b}\left|f(u)-\frac{1}{b-a} \int_{a}^{b} f(t) \Delta t\right|^{s} \Delta u
\end{aligned}
$$

and

$$
\begin{aligned}
K_{2}= & \frac{2}{M-m} \int_{a}^{b}\left[(f(t)-m)(M-f(t))^{2} \log (M-f(t))\right. \\
& \left.+(M-f(t))(f(t)-m)^{2} \log (f(t)-m)\right] \Delta t \\
& +\int_{a}^{b}\left|f(u)-\frac{1}{b-a} \int_{a}^{b} f(t) \Delta t\right|^{2} \log \left|f(u)-\frac{1}{b-a} \int_{a}^{b} f(t) \Delta t\right| \Delta u .
\end{aligned}
$$

Then

(i) for $s>4$,

$$
\frac{\widetilde{\mathcal{D}}_{s}}{s(s-2)} \geq \frac{\widetilde{\mathcal{D}}_{3}}{3}\left(\frac{3 \widetilde{\mathcal{D}}_{4}}{8 \widetilde{\mathcal{D}}_{3}}\right)^{s-3}
$$

(ii) for $1<s<2$,

$$
\frac{\widetilde{\mathcal{D}}_{s}}{s(s-2)} \leq-\widetilde{\mathcal{D}}_{1}\left(-\frac{\widetilde{\mathcal{D}}_{2}}{2 \widetilde{\mathcal{D}}_{1}}\right)^{s-1}
$$


(iii) for $2<s<3$,

$$
\frac{\widetilde{\mathcal{D}}_{s}}{s(s-2)} \leq \frac{\widetilde{\mathcal{D}}_{2}}{2}\left(\frac{2 \widetilde{\mathcal{D}}_{3}}{3 \widetilde{\mathcal{D}}_{2}}\right)^{s-2}
$$

(iv) for $3<s<4$,

$$
\frac{\widetilde{\mathcal{D}}_{s}}{s(s-2)} \leq \frac{\widetilde{\mathcal{D}}_{3}}{3}\left(\frac{3 \widetilde{\mathcal{D}}_{4}}{8 \widetilde{\mathcal{D}}_{3}}\right)^{s-3}
$$

Theorem 5.13. Suppose $p, q \in \mathbb{R}$ such that $1<p<2$ and $\frac{1}{p}+\frac{1}{q}=1$. Let $a, b \in \mathbb{T}$ and $f, g \in \mathrm{C}_{\mathrm{rd}}\left([a, b)_{\mathbb{T}},[m, M]\right)$, where $0 \leq m<M<\infty$, be such that $\int_{a}^{b} g^{q}(t) \Delta t>0$. Then

$$
\begin{aligned}
& \frac{1}{p(p-2)}\left(\left(\int_{a}^{b} g^{q}(t) \Delta t\right)^{p}\left(m^{p}+M^{p}\right)-\left(\int_{a}^{b} g^{q}(t) \Delta t\right)^{p-1} \int_{a}^{b} f^{p}(t) \Delta t\right. \\
& -U_{1}^{p}-\frac{2}{M-m}\left(\int_{a}^{b} g^{q}(t) \Delta t\right)^{p-1} U_{2} \\
& \left.-\int_{a}^{b} g(u) h^{p}(u) \Delta u\left(\int_{a}^{b} g^{q}(t) \Delta t\right)^{p-1}\right) \\
\leq & \frac{1}{2^{p-1}} V_{1}^{2-p}\left(\left(m^{2} \log m+M^{2} \log M\right)\left(\int_{a}^{b} g^{q}(t) \Delta t\right)^{2}\right. \\
& -\int_{a}^{b} g^{q}(t) \Delta t \int_{a}^{b} f^{2}(t) g^{2-q}(t) \log \left(f(t) g^{1-q}(t)\right) \Delta t \\
& -\left((m+M) \int_{a}^{b} g^{q}(t) \Delta t-\int_{a}^{b} f(t) g(t) \Delta t\right)^{2} \\
& \log \left(m+M+\frac{\int_{a}^{b} f(t) g(t) \Delta t}{\int_{a}^{b} g^{q}(t) \Delta t}\right)^{b} \frac{2}{M-m} \int_{a}^{b} g^{q}(t) \Delta t V_{2} \\
& \left.-\int_{a}^{b} g^{q}(t) \Delta t \int_{a}^{b-1} g^{2-q}(u) h^{2}(u) \log \left(g^{1-q}(u) h(u)\right) \Delta u\right)^{p}
\end{aligned}
$$

holds, where

$$
\begin{aligned}
U_{1}= & (m+M) \int_{a}^{b} g^{q}(t) \Delta t-\int_{a}^{b} f(t) g(t) \Delta t \\
U_{2}= & \int_{a}^{b} g^{q}(t)\left(f(t) g^{1-q}(t)-m\right)\left(M-f(t) g^{1-q}(t)\right)^{p} \Delta t \\
& +\int_{a}^{b} g^{q}(t)\left(M-f(t) g^{1-q}(t)\right)\left(f(t) g^{1-q}(t)-m\right)^{p} \Delta t,
\end{aligned}
$$




$$
\begin{aligned}
V_{1}= & \int_{a}^{b} g(u) h(u) \Delta u+\frac{4}{M-m} \int_{a}^{b} g^{q}(t)\left(M-f(t) g^{1-q}(t)\right)\left(f(t) g^{1-q}(t)-m\right) \Delta t \\
V_{2}= & \int_{a}^{b} g^{q}(t)\left[\left(f(t) g^{1-q}(t)-m\right)\left(M-f(t) g^{1-q}(t)\right)^{2} \log \left(M-f(t) g^{1-q}(t)\right)\right. \\
& \left.+\left(M-f(t) g^{1-q}(t)\right)\left(f(t) g^{1-q}(t)-m\right)^{2} \log \left(f(t) g^{1-q}(t)-m\right)\right] \Delta t .
\end{aligned}
$$

Theorem 5.14. Let $\widetilde{\mathcal{J}}_{\Psi_{s}}$ be positive. Then for $r, l, v, w>0$ such that $r \leq v$, $l \leq w$, we have

$$
\widetilde{\mathfrak{M}}_{r, l}^{[s]}(f) \leq \widetilde{\mathfrak{M}}_{v, w}^{[s]}(f)
$$

Theorem 5.15. (i) For all $n \in \mathbb{N}$ and for all $p_{i}>0, p_{i j}=\frac{p_{i}+p_{j}}{2}, 1 \leq$ $i, j \leq n$, the matrix $\left[\widehat{\mathcal{J}}_{\Psi_{p_{i j}}}\right]_{i, j=1}^{n}$ is positive semidefinite;

(ii) the function $s \mapsto \widehat{\mathcal{J}}_{\Psi_{s}}$ is exponentially convex;

(iii) if $\widehat{\mathcal{J}}_{\Psi_{s}}>0$, then the function $s \mapsto \widehat{\mathcal{J}}_{\Psi_{s}}$ is log-convex, i.e., for $0<r<s<$ $w$, we have

$$
\widehat{\mathcal{J}}_{\Psi_{s}}^{w-r} \leq \widehat{\mathcal{J}}_{\Psi_{r}}^{w-s} \widehat{\mathcal{J}}_{\Psi_{w}}^{s-r}
$$

Corollary 5.16. Let $a, b \in \mathbb{T}$ and $f \in \mathrm{C}_{\mathrm{rd}}\left([a, b)_{\mathbb{T}},[m, M]\right)$, where $0 \leq m<M<$ $\infty$. Suppose

$$
\widehat{\mathcal{D}}_{s}= \begin{cases}\frac{M(b-a)-\int_{a}^{b} f(t) \Delta t}{M-m} m^{s}+\frac{\int_{a}^{b} f(t) \Delta t-m(b-a)}{M-m} M^{s} & s \neq 2 \\ -R_{s}-\int_{a}^{b} f^{s}(t) \Delta t, & \\ \frac{M(b-a)-\int_{a}^{b} f(t) \Delta t}{M-m} m^{2} \log m+\frac{\int_{a}^{b} f(t) \Delta t-m(b-a)}{M-m} M^{2} \log M & \\ -R_{2}-\int_{a}^{b} f^{2}(t) \log f(t) \Delta t, & s=2,\end{cases}
$$

where

$$
R_{s}=\frac{1}{M-m} \int_{a}^{b}\left[(f(t)-m)(M-f(t))^{s}+(M-f(t))(f(t)-m)^{s}\right] \Delta t
$$

and

$$
\begin{aligned}
R_{2}= & \frac{1}{M-m} \int_{a}^{b}\left[(f(t)-m)(M-f(t))^{2} \log (M-f(t))\right. \\
& \left.+(M-f(t))(f(t)-m)^{2} \log (f(t)-m)\right] \Delta t
\end{aligned}
$$

Then

(i) for $s>4$,

$$
\frac{\widehat{\mathcal{D}}_{s}}{s(s-2)} \geq \frac{\widehat{\mathcal{D}}_{3}}{3}\left(\frac{3 \widehat{\mathcal{D}}_{4}}{8 \widehat{\mathcal{D}}_{3}}\right)^{s-3}
$$

(ii) for $1<s<2$,

$$
\frac{\widehat{\mathcal{D}}_{s}}{s(s-2)} \leq-\widehat{\mathcal{D}}_{1}\left(-\frac{\widehat{\mathcal{D}}_{2}}{2 \widehat{\mathcal{D}}_{1}}\right)^{s-1}
$$


(iii) for $2<s<3$,

$$
\frac{\widehat{\mathcal{D}}_{s}}{s(s-2)} \leq \frac{\widehat{\mathcal{D}}_{2}}{2}\left(\frac{2 \widehat{\mathcal{D}}_{3}}{3 \widehat{\mathcal{D}}_{2}}\right)^{s-2}
$$

(iv) for $3<s<4$,

$$
\frac{\widehat{\mathcal{D}}_{s}}{s(s-2)} \leq \frac{\widehat{\mathcal{D}}_{3}}{3}\left(\frac{3 \widehat{\mathcal{D}}_{4}}{8 \widehat{\mathcal{D}}_{3}}\right)^{s-3}
$$

Theorem 5.17. Suppose $p, q \in \mathbb{R}$ such that $1<p<2$ and $\frac{1}{p}+\frac{1}{q}=1$. Let $a, b \in \mathbb{T}$ and $f, g \in \mathrm{C}_{\mathrm{rd}}\left([a, b)_{\mathbb{T}},[m, M]\right)$, where $0 \leq m<M<\infty$, be such that $\int_{a}^{b} g^{q}(t) \Delta t>0$. Then

$$
\begin{aligned}
& \frac{1}{p(p-2)}\left(W_{1} m^{p}+W_{2} M^{p}\right. \\
& \left.-\int_{a}^{b} g^{q}(t)\left[\mathfrak{a}(t) \mathfrak{b}^{p}(t)+\mathfrak{b}(t) \mathfrak{a}^{p}(t)\right] \Delta t-(M-m) \int_{a}^{b} f^{p}(t) \Delta t\right) \\
& \leq \frac{1}{2^{2 p-3}}\left(\int_{a}^{b} g^{q}(t) \mathfrak{a}(t) \mathfrak{b}(t) \Delta t\right)^{2-p}\left(W_{1} m^{2} \log m+W_{2} M^{2} \log M\right. \\
& -\int_{a}^{b} g^{q}(t)\left[\mathfrak{a}(t) \mathfrak{b}^{2}(t) \log \mathfrak{b}(t)+\mathfrak{b}(t) \mathfrak{a}^{2}(t) \log \mathfrak{a}(t)\right] \Delta t \\
& \left.-(M-m) \int_{a}^{b} f^{2}(t) g^{2-q}(t) \Delta t\right)^{p-1},
\end{aligned}
$$

holds, where

$$
\begin{gathered}
\mathfrak{a}=f g^{1-q}-m, \quad \mathfrak{b}=M-f g^{1-q} \\
W_{1}=M \int_{a}^{b} g^{q}(t) \Delta t-\int_{a}^{b} f(t) g(t) \Delta t, \quad W_{2}=\int_{a}^{b} f(t) g(t) \Delta t-m \int_{a}^{b} g^{q}(t) \Delta t .
\end{gathered}
$$

Theorem 5.18. Let $\widehat{\mathcal{J}}_{\Psi_{s}}$ be positive. Then for $r, l, v, w>0$ such that $r \leq v$, $l \leq w$, we have

$$
\widehat{\mathfrak{M}}_{r, l}^{[s]}(f) \leq \widehat{\mathfrak{M}}_{v, w}^{[s]}(f)
$$

\section{REFERENCES}

1. S. Abramovich, G. Farid, S. Ivelić and J. Pečarić, More on Cauchy's means and generalization of Hadamard inequality via converses of Jensen's inequality and superquadracity, Int J. Pure Appl. Math. 69 (2011), no. 1, 97-116.

2. S. Abramovich, G. Farid and J. Pečarić, More about Jensen's inequality and Cauchy's means for superquadratic functions, J. Math. Inequal. 7 (2013), no. 1, 11-24.

3. M. Anwar, J. Jakšetić and J. Pečarić, Exponential convexity, positive semi-definite matrices and fundamental inequalities, J. Math. Inequal. 4 (2010), no. 2, 171-189.

4. M. Anwar, R. Bibi, M. Bohner and J. Pečarić, Integral inequalities on time scales via the theory of isotonic linear functionals, Abstr. Appl. Anal. 2011, Art. ID 483595, 16 pages.

5. M. Anwar, R. Bibi, M. Bohner and J. Pečarić, Jensen's functionals on time scales, J. Funct. Spaces Appl. 2012, Art. ID 384045, 17 pages. 
6. J. Barić, R. Bibi, M. Bohner and J. Pečarić, Time scales integral inequalities for superquadratic functions, J. Korean Math. Soc. 50 (2013), no. 3, 465-477.

7. M. Bohner and A. Peterson, Dynamic Equations on Time Scales: An Introduction with Applications, Birkhäuser, Boston, 2001.

8. J.E. Pečarić, F. Proschan and Y.L. Tong, Convex Functions, Partial Orderings, and Statistical Applications, volume 187 of Mathematics in Science and Engineering, Academic Press Inc. Boston, MA, 1992.

${ }^{1}$ School of Natural Sciences, National University of Sciences and TechnolOGY, Islamabad, PAKISTAN.

E-mail address: emaorr@gmail.com; rbibi@camp.nust.edu.pk

2 Department of Mathematics and Statistics, Missouri University of Science And Technology, Rolla, Missouri 65409-0020, USA.

E-mail address: bohner@mst.edu

${ }^{3}$ Faculty of Textile Technology, University of Zagreb, Pierottijeva 6, 10000 Zagreb, Croatia.

E-mail address: pecaric@mahazu.hazu.hr 